\title{
Tuning across Universalities with a Driven Open Condensate
}

\author{
A. Zamora, ${ }^{1}$ L. M. Sieberer, ${ }^{2}$ K. Dunnett, ${ }^{1}$ S. Diehl, ${ }^{3}$ and M. H. Szymańska ${ }^{1,4, *}$ \\ ${ }^{1}$ Department of Physics and Astronomy, University College London, \\ Gower Street, London WC1E 6BT, United Kingdom \\ ${ }^{2}$ Department of Physics, University of California, Berkeley, California 94720, USA \\ ${ }^{3}$ Institute of Theoretical Physics, University of Cologne, D-50937 Cologne, Germany \\ ${ }^{4}$ Kavli Institute for Theoretical Physics, University of California, \\ Santa Barbara, California 93106-4030, USA
}

(Received 13 January 2017; revised manuscript received 12 August 2017; published 13 October 2017)

Driven-dissipative systems in two dimensions can differ substantially from their equilibrium counterparts. In particular, a dramatic loss of off-diagonal algebraic order and superfluidity has been predicted to occur because of the interplay between coherent dynamics and external drive and dissipation in the thermodynamic limit. We show here that the order adopted by the system can be substantially altered by a simple, experimentally viable tuning of the driving process. More precisely, by considering the longwavelength phase dynamics of a polariton quantum fluid in the optical parametric oscillator regime, we demonstrate that simply changing the strength of the pumping mechanism in an appropriate parameter range can substantially alter the level of effective spatial anisotropy induced by the driving laser and move the system into distinct scaling regimes. These include (i) the classic algebraically ordered superfluid below the Berezinskii-Kosterlitz-Thouless (BKT) transition, as in equilibrium; (ii) the nonequilibrium, longwavelength-fluctuation-dominated Kardar-Parisi-Zhang (KPZ) phase; and the two associated topologicaldefect-dominated disordered phases caused by proliferation of (iii) entropic BKT vortex-antivortex pairs or (iv) repelling vortices in the KPZ phase. Furthermore, by analyzing the renormalization group flow in a finite system, we examine the length scales associated with these phases and assess their observability in current experimental conditions.

DOI: 10.1103/PhysRevX.7.041006

Subject Areas: Condensed Matter Physics, Statistical Physics, Superfluidity

\section{INTRODUCTION}

The concept of universality permits us to order and classify a great variety of different physical systems in terms of their common collective long-wavelength behavior close to a critical point. Criticality in systems resting in thermodynamic equilibrium, including its dynamical aspects, is well understood theoretically and has been verified in countless condensed matter experiments [1].

The phenomenon of universality is, however, not bound to the realm of thermodynamic equilibrium and the presence of detailed balance. Paradigmatic examples of nonequilibrium universality are provided by the distinct directed percolation [2,3] and Kardar-Parisi-Zhang universality classes [4]. In contrast to equilibrium, experimental realizations and quantitative observations turn out to be notoriously difficult and, to a large extent, are even elusive to date. The reasons are of practical origin: For example,

*m.szymanska@ucl.ac.uk

Published by the American Physical Society under the terms of the Creative Commons Attribution 4.0 International license. Further distribution of this work must maintain attribution to the author(s) and the published article's title, journal citation, and DOI. the crucial existence of an absorbing state in the directed percolation problem involves a physical fine-tuning of the generic Markovian noise level to zero. The KPZ universality class instead describes the stochastic dynamics of driven interfaces - as present, in principle, in the growth of bacterial colonies [5-7]. Despite its expected ubiquity, so far only specifically designed "KPZ simulators" of liquid crystals in an electric field [8,9] have fully revealed the characteristic scaling behavior quantitatively. Moreover, these experiments are severely limited to one dimension, and two-dimensional experimental platforms where KPZ behavior would have been established unambiguously are already scarce. Promising platforms could be provided by organic and semiconductor thin films [10-14], although further research needs to clarify the possible interference of the probing scheme with the actual KPZ physics [15]. Part of the reason behind this status of affairs lies in the difficulty of creating, in particular, higher-dimensional growing interfaces, which are much less natural than the one-dimensional interfaces, e.g., in combustion of paper [16-18], crystal growth in liquid crystals [8,9], or twodimensional growing films.

Thus, we need more concrete and alternative proposals of experimental platforms to make universal nonequilibrium physics accessible. Previous work has shown that 
two elementary ingredients that are both present in excitonpolariton condensates, routinely produced in semiconductor microcavities $[19,20]$, provide a natural realization of KPZ physics in any dimension, in principle [21-29]. These are (i) a gapless mode, whose existence is protected by the phase-rotation symmetry of the polariton degrees of freedom, and (ii) the breaking of detailed balance on the microscopic level due to the simultaneous presence of reversible coherent dynamics and driven dissipation, realized naturally because of the strong pumping of the systems to ensure a finite many-body excitation density. The role of the growing interface is then played by the phase variable of the driven-open condensate and can be implemented in any dimension, in principle. However, the identification of suitable parameter regimes turns out to be nontrivial also in this class of systems. In particular, in two dimensions, the bottleneck comes from the length scale where macroscopic nonequilibrium effects kick in. This scale is exponentially large unless the breaking of detailed balance is strong.

In this work, we bridge the gap between universal longwavelength nonequilibrium phenomena and the underlying short-distance physics of a concrete experimental platform. We thus provide the so-far missing link between general theory and an actual experimental realization. To this end, we establish parametrically driven microcavities operated in the so-called optical parametric oscillator (OPO) configuration as a suitable platform. In contrast to incoherently pumped exciton-polariton systems, where only an effective mesoscopic description in terms of a stochastic GrossPitaevskii equation with only roughly known parameters can be formulated, the OPO configuration possesses a wellcontrolled and easy-to-manipulate microscopic nature. This comes at the expense of a considerably enhanced complexity microscopically involving three complex modes (referred to as the pump, signal, and idler), but the above requirement of a gapless mode undergoing dynamics with explicitly broken detailed balance is still guaranteed by a residual phase-rotation symmetry for the difference between the signal and idler phases. A key feature is the intrinsic spatial anisotropy of the conventional twodimensional setup rooted in the directionality of the pump drive. Upon varying the pump power and wave vector, we compute the stationary-state phase diagram for this systems. We find that it hosts KPZ physics in viable parameter regimes. In addition, it also lends itself as a platform for other nonequilibrium phenomena related to KPZ universality, such as the possibility of emergent equilibrium behavior at long wavelengths in the case of strong induced spatial anisotropy [30] - a feature that obviously has no counterpart in one-dimensional realizations. From the viewpoint of exciton-polariton systems, our results could thus trigger a targeted search for genuine nonequilibrium phases in OPO systems, which is timely since the accessibility of the equilibrium limit has been demonstrated convincingly in recent years [31,32]. In the following section, we elaborate in more detail on our key results, and provide a guide through the paper.

\section{KEY RESULTS}

The key results of this paper are as follows:

(i) Coherently driven exciton-polaritons are a physical realization of $2 D$ compact anisotropic KPZ physics. This is directly reflected in the experimentally accessible spatial and temporal coherence of the polariton fluid. We establish this connection between the physics of hybrid light-matter systems and growing interfaces in Secs. III A and III B, where we show that at long wavelengths, coherently driven polaritons are described by a 2D compact anisotropic KPZ equation. Consequently, the universal physics in OPO polaritons is much richer than anticipated and has surprising connections to seemingly remote fields such as collective behavior in active systems [30]. The different scaling regimes that are realized in the anisotropic KPZ equation and their manifestation in the polaritons' coherence properties are summarized in Sec. III C: For weak anisotropy, the system falls into the usual (isotropic) KPZ universality class, while for strong anisotropy, the system is described at large scales by a dynamical $X Y$ model.

We note that compactness-i.e., the possible occurrence of topological defects-may hinder the observation of KPZ scaling behavior in the weakly anisotropic regime. However, numerical studies [33] suggest that vortices remain bound in pairs in an extremely long-lived metastable plateau in the case of two-dimensional systems, while they are expected to proliferate in the true stationary state [27,28]. Thus, if there are no vortices in the initial statewhich can always be ensured by suitable postselection-the physics of the KPZ universality class should become accessible.

The universal scaling regimes that can be accessed with polaritons were first discussed in the context of incoherently driven systems in Ref. [21]. However, while in both coherent and incoherent driving schemes the effective long-wavelength theory takes the form of the (anisotropic) KPZ equation, which is the origin of the rich universal behavior, the underlying physics is completely different. In the case of incoherent pumping, the $\mathrm{KPZ}$ equation follows from a standard hydrodynamic description of the dynamics of the polariton fluid and governs fluctuations of the phase of the condensate. On the other hand, coherent laser driving pins the condensate phase at the pump wave vector, and the derivation of the long-wavelength theory is much more subtle: Here, it is the relative phase of the signal and idler modes, which are both 
macroscopically populated above the OPO threshold, that is free to fluctuate. This is the Goldstone mode that determines the physics at large scales. The drive term also explicitly breaks rotational symmetry. Thus, we naturally have spatial anisotropy in the system, which can be easily tuned over a wide range.

(ii) We perform first-principles computations of the parameters of the long-wavelength KPZ equation. Thus, experimental and numerical investigations can precisely target interesting regimes. To this end, we capitalize on the well-controlled nature of the microscopic dynamics. This is in stark contrast to incoherently driven polaritons, for which the parameters entering the commonly employed phenomenological description [19] are known only approximately and no accurate microscopic description exists. Moreover, as a consequence of the different mechanisms leading to the KPZ equation, the natural scales of the coefficients appearing in this equation are vastly different in incoherently and coherently pumped polaritons. In particular, in the former case, both the characteristic KPZ nonlinearity and the degree of anisotropy are typically small [21], making the observation of novel nonequilibrium features related to that property challenging. In contrast, here we show that in OPO systems, those quantities can be tuned over a wide range of values simply by changing the driving strength, the pump wave vector, and the detuning between photons and excitons-which is easily done in experiments. Hence, we determine under which conditions these scaling regimes are feasible in current experiments on inorganic and organic microcavities. Moreover, we analyze the renormalization group (RG) flow equations in a finite system and estimate the relevant length scales by varying the control parameters such as the external laser drive and the detuning between the cavity photons and the excitons. In particular, we find a parameter range in which the $\mathrm{KPZ}$ nonlinearity is so strong that we would expect KPZ scaling to set in even on very short scalesprovided this strongly nonlinear regime is not destabilized by other types of fluctuations.

The renormalization of parameters and the resulting scaling behavior of correlation functions are fundamentally induced by strong fluctuations, making the mean-field approach, which has been applied in most of the existing literature on polaritons in the OPO regime [34-36], insufficient. Rather, the RG techniques we use are tailored to address universal behavior that occurs on large length and time scales, beyond what is accessible with exact numerical methods [33].

(iii) As a consequence of the exquisite tunability, we show that different universal scaling regimes can easily be reached with a single experimental setup.
Thus, our work sheds further light on the fundamental question of which types of order and phase transitions can be realized in these systems; at present, this is still subject to an intense debate. This is particularly true in light of the fact that exact stochastic simulations able to account for vortices [33], but also experiments [37], observed a clear transition from exponential decay of correlations to algebraic order but with an algebraic exponent $\alpha$ as large as 4 times the equilibrium upper bound, when approaching the BKT transition, suggesting an "overshaken" superfluid state [33].

As we show, the type of order adopted is not, in fact, an intrinsic property of the system, but it can be strongly sensitive to the driving process that is able to tune the system between two different universality classes by only a relatively small change in the driving strength. Let us emphasize again that the key feature we exploit here is the spatial anisotropy that is imprinted on the system by the wave vector of the driving laser. In the long-wavelength theory for parametrically driven polaritons, the effective degree of anisotropy is measured by a single parameter $\Gamma$. This quantity depends, in a nontrivial way, on the system parameters and, in particular, on the driving strength. As we discuss in Sec. IV, in the region close to the OPO upper threshold, but at lower powers, the system develops a steady state, which (if vortices remain bound) falls into the KPZ universality class $[4,21]$ with a nonequilibrium fixed point $[21,30]$ and no counterpart in equilibrium systems. However, by increasing the strength of the external drive towards the OPO upper threshold, the effective anisotropy crosses a critical value, and the properties of the system are governed by an equilibrium fixed point corresponding to a dynamical $X Y$ model. Then, algebraic order and superfluidity can be established through the usual BKT transition.

The equilibrium fixed point is approached for larger driving strengths than the nonequilibrium one, suggesting that we are not simply observing an approach to equilibrium as the external dissipation diminishes, but rather a more profound interplay between drive, dissipation, and spatial anisotropy. In fact, since spatial anisotropy is a sine qua non, this mechanism is profoundly different from the asymptotic thermalization that has been discussed in a variety of systems [38-48].

\section{SYSTEM AND THEORETICAL DESCRIPTION}

Exciton-polaritons are bosonic quasiparticles emerging in the regime of strong coupling between excitons in semiconductors and a cavity photon mode [19,20] (see Fig. 1). The coherent mixing between light and matter excitations results in the emergence of two bands in these systems, termed the upper and lower polaritons. Because of mirror imperfections, the lifetime of photons and hence 


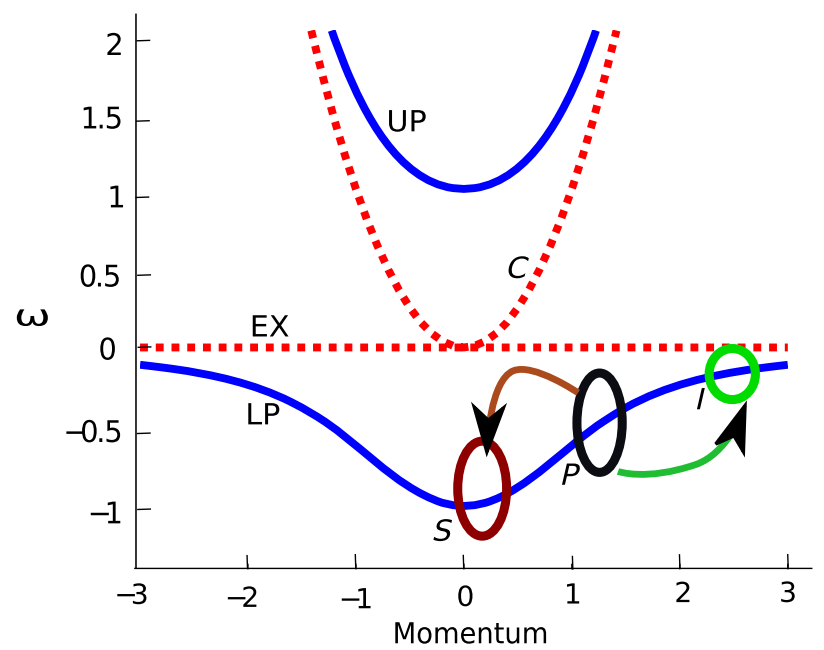

FIG. 1. Polariton system. Dispersions for the exciton (EX) and cavity photon $(C)$ with zero detuning mixing to form upper (UP) and lower polaritons (LP). The external drive introduces pairs of lower polaritons with momentum $\mathbf{k}_{p}$ and energy $\omega_{p}(P)$, which scatter into the signal $(S)$ and idler $(I)$ states while conserving energy and momentum.

polaritons is finite, necessitating continuous external laser driving to maintain their finite population. In the stationary state resulting from the compensation of gains and losses, detailed balance is violated, and the system is therefore not in thermal equilibrium.

The properties of this nonequilibrium stationary state are vitally influenced by the implementation of the laser driving. In particular, if the laser frequency is chosen to resonantly populate highly excited states, the polaritons generated in this way undergo complex scattering before condensing in the lower polariton band. All coherence of the exciting laser is lost in these processes and not transferred to the lower polariton states. The dynamics of the incipient condensate under such incoherent pumping is commonly described phenomenologically in terms of a generalized Gross-Pitaevskii equation [19]. In contrast to the incoherent pumping scheme, in the coherent scheme, polaritons are excited with a monochromatic external laser acting resonantly on or close to the lower polariton dispersion [49]. The absence of complex scattering processes facilitates an $a b$ initio rather than a phenomenological description, as we detail below. As a consequence of coherent driving of lower polaritons, two symmetries that are generically present for incoherent pumping should be discussed in some more detail at this point: (i) $U(1)$ symmetry under rotations of the phase of the lower polariton field and (ii) symmetry under spatial rotations.

Point (ii) is addressed by specifying the coherent driving term for our problem, $f_{p}^{*} \psi_{p}+$ H.c., with external force $f_{p}=F_{p} e^{i\left(\mathbf{k}_{p} \cdot \mathbf{r}-\omega_{p} t\right)}$ and pump field $\psi_{p}$. Clearly, the directionality imprinted by the external driving field explicitly breaks the rotational symmetry of the problem on the microscopic level. It is a key point of this paper to elaborate on the consequences of this fact for the macroscopic observables.

Regarding point (i), we need to specify the interaction term between the pump field $\psi_{p}$, and the signal and idler modes $\psi_{s}, \psi_{i}$, respectively. It describes the interconversion of two pump field photons into a pair of signal and idler photons (see Fig. 1), $\sim \psi_{i}^{*} \psi_{s}^{*} \psi_{p}^{2}+$ H.c. Thus, we have three phases of pump, signal, and idler fields. However, the external coherent pump term locks the phase of the pump field $\psi_{p}$ via the coherent drive term, in turn locking the sum of phases of signal and idler fields $\psi_{s}$ and $\psi_{i}$ via the interaction term. On the other hand, their difference is not fixed by the dynamics of the system. Thus, there is one remaining $U(1)$ phase-rotation invariance left, which is generated by the transformation

$$
\left(\begin{array}{c}
\psi_{s} \\
\psi_{i} \\
\psi_{p}
\end{array}\right) \mapsto\left(\begin{array}{ccc}
e^{i \alpha} & 0 & 0 \\
0 & e^{-i \alpha} & 0 \\
0 & 0 & 1
\end{array}\right)\left(\begin{array}{c}
\psi_{s} \\
\psi_{i} \\
\psi_{p}
\end{array}\right) .
$$

The residual $U(1)$ symmetry introduced in this way can be broken spontaneously and is responsible for the existence of a gapless phase mode above the OPO threshold [46,50-52], which in turn governs the long-distance coherence behavior of the OPO condensate to be investigated in this paper.

More precisely, in a coherently driven system, depending on the strength of the external pump power, we distinguish between two different regimes: the pump-only state, with only one mode, $\psi_{p}$, substantially occupied (white region in Fig. 2). Such a state is characterized by a momentum $\mathbf{k}_{p}$ and frequency $\omega_{p}$, which coincide with the momentum and frequency of the external pump. The phase of the pumponly state is locked by the external drive. However, in a certain pumping regime (green region in Fig. 2), pairs of polaritons scatter from the pump state into two new substantially occupied states, the signal $\psi_{s}$ and the idler $\psi_{i}$, with momentum $\mathbf{k}_{s}$ and $\mathbf{k}_{i}$ and frequencies $\omega_{s}$ and $\omega_{i}$, respectively (see Fig. 1). The scattering process is determined by the resonance conditions: $\mathbf{k}_{s}+\mathbf{k}_{i}=2 \mathbf{k}_{p}$ and $\omega_{s}+\omega_{i}=2 \omega_{p}$. Thus, in the OPO regime, the lower polariton field $\psi_{\mathrm{LP}}$ can be split into three contributions:

$$
\psi_{\mathrm{LP}}(\mathbf{r}, t)=\sum_{j=s, p, i} \psi_{j}(\mathbf{r}, t) e^{i\left(\mathbf{k}_{j} \cdot \mathbf{r}-\omega_{j} t\right)} .
$$

In a mean-field treatment ignoring fluctuations, the amplitudes $\psi_{j}(\mathbf{r}, t)$ are spatially homogeneous and time independent. Below, we study the influence of fluctuations on the spatial and temporal coherence properties of the lower polariton field.

The yellow region in Fig. 2 marks the range of parameters in which polaritons are parametrically scattered to more than two additional momentum states (i.e., there 


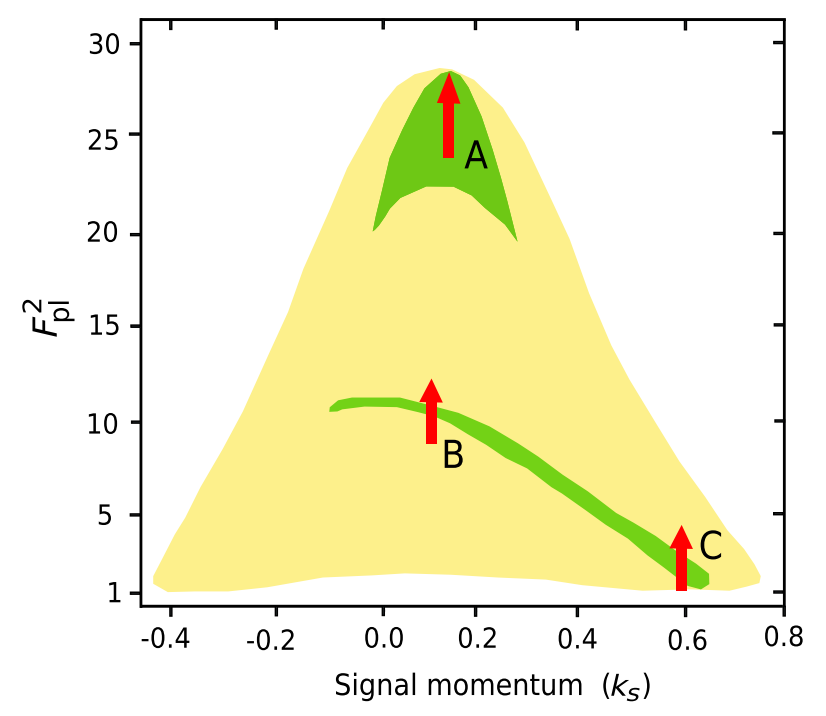

FIG. 2. Stability diagram. Regions of instability of the pumponly state towards an OPO solution (yellow and green). The simplest three-mode OPO state is stable for parameters marked by green, i.e., at $k_{s}$ around 0.1 for high $F_{p}^{2}$ (regions $\mathrm{A}$ and $\mathrm{B}$ ) and over a more extended higher $k_{s}$ range at low $F_{p}^{2}$ (region $\mathrm{C}$ ). In the yellow region, both the pump-only and the three-mode ansatz are unstable. The red arrows indicate the direction of increasing pump power shown in Figs. 4-6. Note that the pump intensity (vertical axis) is normalized to the lower threshold, $F_{p l}^{2} \equiv$ $\left(F_{p} / F_{p}^{\text {lo }}\right)^{2}$. Parameters are the same as in the text with zero detuning and $k_{p}=1.4$.

are additional satellite states or the signal mode extends over a ring in momentum space). However, here we focus on the regime in which the three-mode ansatz (2) is stable.

As a short digression, it is interesting to note which kind of order is established upon crossing the OPO threshold. Assuming that the amplitudes in Eq. (2) are constants, which we write in the density-phase representation as $\psi_{j}=\sqrt{\rho_{j}} e^{i \phi_{j}}$, the density of lower polaritons is given by

$$
\begin{aligned}
\left|\psi_{\mathrm{LP}}(\mathbf{r}, t)\right|^{2}= & \sum_{j} \rho_{j}^{2} \\
& +2\left(\sqrt{\rho_{s} \rho_{p}} \cos \left(\phi_{s}-\phi_{p}+\left(\mathbf{k}_{s i} \cdot \mathbf{r}-\omega_{s i} t\right) / 2\right)\right. \\
& +\sqrt{\rho_{p} \rho_{i}} \cos \left(\phi_{i}-\phi_{p}-\left(\mathbf{k}_{s i} \cdot \mathbf{r}-\omega_{s i} t\right) / 2\right) \\
& \left.+\sqrt{\rho_{s} \rho_{i}} \cos \left(\phi_{s}-\phi_{i}+\mathbf{k}_{s i} \cdot \mathbf{r}-\omega_{s i} t\right)\right), \quad(3)
\end{aligned}
$$

where we denote $\mathbf{k}_{s i}=\mathbf{k}_{s}-\mathbf{k}_{i}$ and $\omega_{s i}=\omega_{s}-\omega_{i}$, and we use the resonance conditions stated above. Thus, at the mean-field level, the OPO regime is characterized by density-wave and time-crystal order, with base wave vector and frequency $\mathbf{k}_{s i} / 2$ and $\omega_{s i} / 2$, respectively. The Goldstone mode alluded to above corresponds to fluctuations of the relative phase $\phi_{s}-\phi_{i}$. These can be incorporated in Eq. (3) by replacing $\phi_{s} \rightarrow \phi_{s}+\theta(\mathbf{r}, t)$ and $\phi_{i} \rightarrow \phi_{i}-\theta(\mathbf{r}, t)$ (the analysis of fluctuations is carried out systematically below), which shows that the Goldstone mode is encoded in the phase of the spatiotemporally periodic order. In particular, topological defects in $\theta(\mathbf{r}, t)$ are dislocations in the combined density wave and time crystal. However, by filtering the lower polariton field in momentum space as is usually done in experiments [53], it is possible to single out the signal and idler modes. Then, topological defects in $\theta(\mathbf{r}, t)$ appear as ordinary vortices. Using this approach, coherence of the OPO state can be quantified by measuring the two-point correlation function of the signal mode,

$$
g^{(1)}(\mathbf{r}, t)=\left\langle\psi_{s}(\mathbf{r}, t) \psi_{s}^{*}(0,0)\right\rangle .
$$

In the following, we investigate how mean-field order is affected by fluctuations of the Goldstone mode.

\section{A. Keldysh field integral approach}

As pointed out above, for coherently pumped polaritons, it is possible to derive a microscopic description. A convenient framework is provided by the Keldysh field integral formalism (see Ref. [26] for a recent review on applications to driven-dissipative systems). In this formalism, the coupled dynamics of excitons and photons under the influence of laser driving and cavity losses is encoded in a field integral [54] $Z=\int \mathcal{D}\left[\psi_{s}, \psi_{i}, \psi_{p}, \bar{\psi}_{s}, \bar{\psi}_{i}, \bar{\psi}_{p}\right] e^{i S_{\mathrm{OPO}}}$, which we write here already in terms of the three polariton modes introduced in Eq. (2). Detailed balance conditions are explicitly violated by the pumping and loss terms [55]. Since we are interested in the long-range dynamics of the system, we neglect quartic terms involving more than a single quantum field. This semiclassical approximation is applicable in the long-wavelength limit and can be justified formally by canonical power counting, which shows that the neglected terms are irrelevant in the renormalization group sense [25]. Thus, the action $S_{\mathrm{OPO}}$ becomes $S_{C}$ :

$$
\begin{aligned}
S_{C}= & \int d t d^{2} \mathbf{r}\left\{-\left(f_{p}^{*} \psi_{p}^{Q}+f_{p} \bar{\psi}_{p}^{Q}\right)\right. \\
& +\sum_{j=s, p, i}\left[\frac{1}{X_{j}^{2}}\left(\bar{\psi}_{j}^{C} \bar{\psi}_{j}^{Q}\right)\left(\begin{array}{cc}
0 & {\left[D_{0}^{A}\right]_{j}^{-1}} \\
{\left[D_{0}^{R}\right]_{j}^{-1}} & {\left[D_{0}^{-1}\right]_{j}^{K}}
\end{array}\right)\left(\begin{array}{c}
\psi_{j}^{C} \\
\psi_{j}^{Q}
\end{array}\right)\right. \\
& \left.-g_{X}\left(\left(2\left(\left|\psi_{s}^{C}\right|^{2}+\left|\psi_{i}^{C}\right|^{2}+\left|\psi_{p}^{C}\right|^{2}\right)-\left|\psi_{j}^{C}\right|^{2}\right) \psi_{j}^{C} \bar{\psi}_{j}^{Q}+\text { c.c. }\right)\right] \\
& \left.-g_{X}\left(2 \psi_{s}^{C} \psi_{i}^{C} \bar{\psi}_{p}^{C} \bar{\psi}_{p}^{Q}+\left(\psi_{p}^{C}\right)^{2}\left(\bar{\psi}_{i}^{C} \bar{\psi}_{s}^{Q}+\bar{\psi}_{s}^{C} \bar{\psi}_{i}^{Q}\right)+\text { c.c. }\right)\right\},
\end{aligned}
$$

where $\psi_{c, j}\left(\psi_{q, j}\right)$ is the classical (quantum) component of the field $\psi_{j}$. The inverse advanced Green's function reads $\left[D_{0}^{A}\right]_{j}^{-1}=i \partial_{t}+\omega_{j}-\omega_{\mathrm{LP}}\left(\mathbf{k}_{j}-i \nabla\right)-i \gamma_{j}$, from which the inverse retarded Green's function can be obtained by using the relation $\left[D_{0}^{R}\right]_{j}^{-1}=\left(\left[D_{0}^{A}\right]_{j}^{-1}\right)^{\dagger} ; \gamma_{j}$ is the decay rate of the mode $j$, and the lower polariton dispersion, in dimensionless units, [56] is given by 


$$
\omega_{\mathrm{LP}}(\mathbf{q})=\frac{1}{2}\left(q^{2}+\delta_{C X}-\sqrt{\left(q^{2}+\delta_{C X}\right)^{2}+4}\right),
$$

where $\delta_{C X}$ is the detuning between cavity photons and excitons. A typical dispersion relation for zero detuning is shown in Fig. 1. The Keldysh part of the inverse Green's function, $\left[D_{0}^{-1}\right]_{j}^{K}=i 2 \gamma_{j}$, stems from integrating out the bosonic decay bath fields in the Markovian approximation [54]. Here, $X_{j} \equiv X\left(\mathbf{k}_{j}\right)$ is the excitonic Hopfield coefficient of the mode $j$ with momentum $\mathbf{k}_{j}$ [20], and $g_{X}$ is the strength of the exciton-exciton interaction. Finally, the monochromatic external pump is, as mentioned previously, of the form $f_{p}=F_{p} e^{i\left(\mathbf{k}_{p} \cdot \mathbf{r}-\omega_{p} t\right)}$, where $F_{p}$ is taken to be a positive real number. Since the action $S_{C}$ is only quadratic in quantum fields, we can make use of the Martin-SiggiaRose formalism $[25,57,58]$ and map the functional integral to the set of coupled stochastic differential equations for the signal, idler, and pump modes, which determine the dynamics of the system:

$$
\begin{aligned}
i \partial_{t} \psi_{s} & =\Omega_{s} \psi_{s}-i \gamma_{s}+\tilde{g}_{X} \psi_{p}^{2} \psi_{i}^{*}+\xi_{s}, \\
i \partial_{t} \psi_{i} & =\Omega_{i} \psi_{i}-i \gamma_{i}+\tilde{g}_{X} \psi_{p}^{2} \psi_{s}^{*}+\xi_{i}, \\
i \partial_{t} \psi_{p} & =\Omega_{p} \psi_{p}-i \gamma_{p}+2 \tilde{g}_{X} \psi_{s} \psi_{i} \psi_{p}^{*}+f_{p}+\xi_{p},
\end{aligned}
$$

where $\tilde{g}_{X} \equiv g_{X} X_{p}^{2} X_{i} X_{s}$ and $\psi_{j} \equiv \psi_{j} / X_{j}$. We use the shorthand notation [54] $\Omega_{j} \equiv-\omega_{j}+\omega_{\mathrm{LP}}\left(\mathbf{k}_{j}-i \nabla\right)+$ $g_{X} X_{j}^{2}\left(2\left(\tilde{n}_{s}+\tilde{n}_{p}+\tilde{n}_{i}\right)-\tilde{n}_{j}\right)\left(\right.$ with $\left.\tilde{n}_{j}=X_{j}^{2}\left|\psi_{j}\right|^{2}\right)$, and we redefine the external pump $X_{p} f_{p} \rightarrow f_{p}$. The terms $\xi_{s, i, p}$ are Gaussian noise sources that have a vanishing expectation value, $\left\langle\xi_{j}(\mathbf{r}, t)\right\rangle=0$, and a white spectrum, $\left\langle\xi_{j}(\mathbf{r}, t) \xi_{j^{\prime}}^{*}\left(\mathbf{r}^{\prime}, t^{\prime}\right)\right\rangle=2 \gamma_{j} \delta\left(\mathbf{r}-\mathbf{r}^{\prime}\right) \delta\left(t-t^{\prime}\right) \delta_{j j^{\prime}}$. Note that the dynamical equations (7) are invariant under the $U(1)$ transformation expressed in Eq. (1).

\section{B. Long-wavelength theory in the OPO regime: Mapping to the anisotropic KPZ equation}

The stochastic equations (7) for the signal, idler, and pump modes provide a convenient starting point for deriving the effective long-wavelength theory for polaritons in the OPO regime. We follow the usual strategy of parametrizing fluctuations around the mean-field solution in the density-phase representation; i.e., we write the three modes as

$$
\psi_{j}(\mathbf{r}, t)=\left(\sqrt{\rho_{j}}+\pi_{j}(\mathbf{r}, t)\right) e^{i\left(\phi_{j}+\theta_{j}(\mathbf{r}, t)\right)},
$$

where $\sqrt{\rho_{j}}$ and $\phi_{j}$ are the homogeneous and stationary mean-field density and phase, respectively, obtained by solving Eq. (7) with $\xi_{s, i, p} \equiv 0$; fluctuations around the mean-field solution are encoded in the fields $\pi_{j}(\mathbf{r}, t)$ and $\theta_{j}(\mathbf{r}, t)$. The key point that allows us to considerably simplify the equations resulting from inserting the ansatz (8) in Eq. (7) is that fluctuations of the relative phase $\theta(\mathbf{r}, t)=\theta_{s}(\mathbf{r}, t)-\theta_{i}(\mathbf{r}, t)$ of signal and idler modes, i.e., fluctuations of the Goldstone mode, are gapless because of the $U(1)$ symmetry expressed in Eq. (1), while fluctuations of all others are gapped. This implies that in the limit of long wavelengths and low frequencies, the latter fluctuations are small, and the equations of motion can be linearized in these variables, which can then be eliminated. Details of this calculation are given in Appendix A. This results in a single stochastic equation for the Goldstone mode $\theta$, which takes the form of the anisotropic KPZ (aKPZ) [30,59] equation with an additional drift term proportional to $\nabla \theta$ :

$$
\partial_{t} \theta=\sum_{i=x, y}\left[D_{i} \partial_{i}^{2} \theta+\frac{\lambda_{i}}{2}\left(\partial_{i} \theta\right)^{2}\right]+\mathbf{B} \cdot \nabla \theta+\eta .
$$

The coefficients $D_{x, y}, \lambda_{x, y}$, and $\mathbf{B}$ result from linear combinations of different parameters appearing in Eqs. (7) (see Appendix A for details). The Gaussian white noise term $\eta$ derives from the different noise terms $\xi_{j}$ and satisfies $\langle\eta(\mathbf{r}, t)\rangle=0$ and $\left\langle\eta(\mathbf{r}, t) \eta\left(\mathbf{r}^{\prime}, t^{\prime}\right)\right\rangle=2 \Delta \delta\left(\mathbf{r}-\mathbf{r}^{\prime}\right) \delta\left(t-t^{\prime}\right)$, where the noise strength $\Delta$ is related to the decay rates $\gamma_{j}$. In addition to eliminating massive fluctuations as discussed above, to obtain Eq. (9) we also expanded the lower polariton dispersion (6) around each mode $j$ with momentum $\mathbf{k}_{j}$ to second order in the gradient:

$$
\omega_{\mathrm{LP}}\left(\mathbf{k}_{j}-i \nabla\right) \approx \omega_{\mathrm{LP}}\left(\mathbf{k}_{j}\right)-i \omega_{1 j} \cdot \nabla-\nabla^{T} \omega_{2 j} \nabla .
$$

This expression shows clearly that the finite value of the pump wave vector $\mathbf{k}_{p}$ (and hence of the idler wave vector, and, in some cases, also of the signal wave vector) lies at the heart of the spatial anisotropy of the system, which leads, in particular, to the matrix $\omega_{2 j}$ having two distinct eigenvalues, resulting in $D_{x} \neq D_{y}$ and $\lambda_{x} \neq \lambda_{y}$ in the effective long-wavelength description, Eq. (9). This should be compared to incoherently pumped polaritons, for which the expansion of the lower polariton dispersion around zero momentum, $\omega_{\mathrm{LP}}(-i \nabla) \approx \omega_{\mathrm{LP}}(0)-\nabla^{2} /\left(2 m_{\mathrm{LP}}\right)$, where $m_{\mathrm{LP}}$ is the mass of lower polaritons, leads to the isotropic $\mathrm{KPZ}$ equation $[21,60]$. Below, we show how the values $D_{x, y}$ and $\lambda_{x, y}$, and hence the effective degree of anisotropy, depend on system parameters such as pumping and detuning. Crucially, by tuning these parameters, we can access different universal scaling regimes of Eq. (9).

We note that the drift term $\mathbf{B} \cdot \nabla \theta$ can be eliminated from Eq. (9) by introducing a new variable $\theta^{\prime}(\mathbf{r}, t)=$ $\theta\left(\mathbf{r}+\mathbf{v}_{0} t, t\right)$, i.e., by transforming to a frame of reference that moves at a velocity $\mathbf{v}_{0}$. For $\mathbf{v}_{0}=\mathbf{B}$, the equation of motion of $\theta^{\prime}$ is given by the aKPZ equation without the drift term. It is thus sufficient to consider the latter equation and transform back to the laboratory frame of reference only for calculating observables in terms of the original variable $\theta$. 


\section{Scaling regimes of the anisotropic KPZ equation}

In the previous section, we showed that fluctuations around the three-mode OPO state (2) are governed by the anisotropic KPZ equation (9). What does this mean for the spatial and temporal coherence of the polariton condensate as measured by the first-order coherence function Eq. (4)? There are three aspects that make the physics of Eq. (9) rich but also complex to analyze: (i) spatial anisotropy, (ii) the nonlinear terms with coefficients $\lambda_{x, y}$, and (iii) the compactness of $\theta$, which implies that this field can contain topological defects. Approaching the problem analytically, difficulties (ii) and (iii) can be controlled perturbatively, if both the nonlinearities $\lambda_{x, y}$ and the vortex fugacity $y$, which is a measure of the probability of vortex-antivortex pairs forming at a microscopic distance, are small parameters [62]. Then, as we describe in the following, depending on the strength of anisotropy quantified by the anisotropy parameter

$$
\Gamma \equiv \lambda_{y} D_{x} /\left(\lambda_{x} D_{y}\right),
$$

based on the perturbative treatment, we expect strikingly different behavior in the weakly and strongly anisotropic regimes, characterized by $\Gamma>0$ and $\Gamma<0$, respectively.

To understand why $\Gamma=0$ separates these regimes, we first note that $D_{x, y}>0$ is required for Eq. (9) to be dynamically stable; therefore, $\Gamma<0$ corresponds to $\lambda_{x}$ and $\lambda_{y}$ having opposite signs. If $\lambda_{x}$ and $\lambda_{y}$ have the same sign and hence $\Gamma>0$, it does not make a difference whether $\lambda_{x, y}$ are both positive or negative. In fact, the former case is related to the latter by the transformation $\theta \rightarrow-\theta$ in Eq. (9) (after the drift term has been removed as described above). Thus, the physics can change qualitatively only when $\lambda_{x, y}$ have opposite signs, and thus $\Gamma<0$. This is indeed found to be the case in the RG analysis.

In the weakly anisotropic (WA) regime, both the nonlinear terms $\lambda_{x, y}[30,59]$ and the fugacity $y[27]$ are relevant couplings; i.e., they grow under renormalization. In the absence of vortices, this would imply that the correlation function $g^{(1)}(\mathbf{r}, t)$ takes the form of a stretched exponential with KPZ scaling exponents [see Eq. (14) below]. This behavior would be observable on length and time scales greater than $L_{\mathrm{KPZ}}$ and $t_{\mathrm{KPZ}}$, respectively, which mark the breakdown of the perturbative treatment in $\lambda_{x, y}$. However, eventually, vortices might unbind at a scale $L_{v}$ and after a time $t_{v}$ [27], leading to exponential decay of correlations (and an absence of superfluid behavior) beyond these scales. For a detailed discussion of the influence of vortices in this regime, see Appendix B.

The physics is quite different in the strongly anisotropic (SA) regime: For $\Gamma<0$, the nonlinearities $\lambda_{x, y}$ are irrelevant and flow to zero. Then, the linearized version of Eq. (9) exhibits a BKT transition driven by the noise strength (which in turn depends on the loss rates and the external drive; see Appendix A); i.e., at low noise, a superfluid phase with algebraic order is possible $[21,30]$ even in the thermodynamic limit of an infinite system. Intriguingly, OPO polaritons allow us to cross the boundary between the WA and SA regimes. This is shown in the next section. In the remainder of the present section, we discuss in detail the RG flow and the two scaling regimes of the aKPZ equation.

The RG flow of the aKPZ equation in the absence of vortices was analyzed in Refs. [30,59]. It can be parametrized in terms of only two independent quantities - the anisotropy parameter $\Gamma$ introduced in Eq. (11) and the rescaled dimensionless nonlinearity $g$, which is defined as

$$
g \equiv \lambda_{x}^{2} \Delta /\left(D_{x}^{2} \sqrt{D_{x} D_{y}}\right) .
$$

To leading order in $g$, the RG flow equations read

$$
\begin{aligned}
& \frac{d g}{d l}=\frac{g^{2}}{32 \pi}\left(\Gamma^{2}+4 \Gamma-1\right), \\
& \frac{d \Gamma}{d l}=\frac{\Gamma g}{32 \pi}\left(1-\Gamma^{2}\right) .
\end{aligned}
$$

As described above, depending on the value of $\Gamma$, we distinguish between WA and SA regimes. In the former case, $\lambda_{x}$ and $\lambda_{y}$ have the same sign (the coefficients $D_{x, y}$ have to be positive to ensure stability). Then, the nonlinearity is marginally relevant, and the RG flow takes the system to a strong coupling fixed point at $g_{*}$, which is beyond the scope of the perturbative treatment. Moreover, $\Gamma \rightarrow 1$; i.e., at large scales, rotational symmetry is restored, [63] and thus the system falls into the usual isotropic KPZ universality class.

On the other hand, in the SA regime with $\Gamma<0$, which is realized when the coefficients $\lambda_{x}$ and $\lambda_{y}$ have opposite sign, the nonlinearity $g$ is irrelevant and flows to zero. As a consequence, the aKPZ equation becomes a linear stochastic differential equation, which is governed by an equilibrium fixed point at $g=0, \Gamma=-1$, and the system falls into the (dynamical) $X Y$ universality class.

Having discussed how the RG flow of the aKPZ equation is structured by different fixed points in the WA and SA regimes, it is natural to ask for observable consequences of these findings. Universal scaling behavior leaves its mark in the long-time and long-range decay of correlations. Hence, in the following, we discuss the form of the correlation function (4) implied by these results, as well as which modifications are to be expected because of the possible occurrence of vortices.

\section{Weakly anisotropic regime}

In the WA regime, the two-point correlation function (4), $g^{(1)}(\mathbf{r}, t) \propto e^{-C(\mathbf{r}, t) / 2}$ (this form assumes that density fluctuations are negligible as compared to fluctuations of the Goldstone mode; see Sec. III B), where $C(\mathbf{r}, t)=$ $\left\langle(\theta(\mathbf{r}, t)-\theta(0,0))^{2}\right\rangle$, shows a stretched exponential decay [64]: 
$C(\mathbf{r}, t) \sim \tilde{r}^{2 \chi} F_{\mathrm{KPZ}}\left(c_{1} t / \tilde{r}^{z}\right) \sim \begin{cases}\tilde{r}^{2 \chi} & \text { for } \tilde{r}^{z} \gg c_{1} t \\ t^{2 \chi / z} & \text { for } c_{1} t \gg \tilde{r}^{z},\end{cases}$

where $\tilde{r}^{2}=\left(x / x_{0}\right)^{2}+\left(y / y_{0}\right)^{2}$ encodes the anisotropy of the system and the parameter $c_{1}$ depends on the microscopic parameters. The limiting forms follow from the asymptotic behavior of the scaling function, $F_{\mathrm{KPZ}}(w) \sim A_{1}$ for $w \rightarrow 0$ and $F_{\mathrm{KPZ}}(w) \sim A_{2} w^{2 \chi / z}$ for $w \rightarrow \infty$, with nonuniversal constants $A_{1}$ and $A_{2}$. In two spatial dimensions, the roughness exponent is $\chi \approx 0.39$ (see Refs. $[13,65]$ for recent numerical investigations of KPZ scaling and Ref. [66] for a functional RG analysis), and the dynamical exponent $z$ can be obtained from the exact scaling relation $\chi+z=2$.

The scaling form Eq. (14) applies to the comoving reference frame [see the discussion below Eq. (9)], in which the drift term $\mathbf{B} \cdot \nabla \theta$ is absent. We can calculate $g^{(1)}(\mathbf{r}, t)$ in the original frame simply by replacing $\mathbf{r} \mapsto \mathbf{r}+\mathbf{B} t$ in Eq. (14), which yields

$$
C(\mathbf{r}, t) \sim|\mathbf{r}+\mathbf{B} t|^{2 \chi} F_{\mathrm{KPZ}}\left(c_{1} t /|\mathbf{r}+\mathbf{B} t|^{z}\right) .
$$

From this expression, we explore the consequences of a nonvanishing drift term $\mathbf{B}$ on the correlations of the system for the KPZ scaling. In particular, we find, for spatial correlations at equal times,

$$
C^{\prime}(\mathbf{r}, 0) \sim \tilde{r}^{2 \chi},
$$

which coincides with the result for the case of a vanishing drift term. However, temporal correlations are modified:

$$
\begin{aligned}
C^{\prime}(0, t) & \sim(B t)^{2 \chi} F_{\mathrm{KPZ}}\left(c_{1} t /(B t)^{z}\right) \\
& \sim \begin{cases}t^{2 \chi / z} & \text { for } t \ll \tau_{c} \\
t^{2 \chi} & \text { for } t \gg \tau_{c} .\end{cases}
\end{aligned}
$$

Hence, the system exhibits two different exponents, depending on the time scale. Initially, the correlator $g^{(1)}(0, t)$ shows stretched exponential decay with exponent $2 \chi / z$, characteristic of KPZ scaling. At longer times, the drift term causes the exponent to increase to $2 \chi$, resulting in a faster decay of temporal correlations. The crossover time $\tau_{c}$ at which the transition between the two regimes occurs can be obtained from $c_{1} \tau_{c} \sim\left(B \tau_{c}\right)^{z}$, leading to $\tau_{c} \sim\left(B^{z} / c_{1}\right)^{1 /(1-z)}$. The scaling forms (16) and (17) are approached on certain length and time scales. For small KPZ nonlinearity $g$, the scale $L_{\mathrm{KPZ}}$ above which spatial correlations are expected to behave as Eq. (16) can, in the isotropic case, be estimated as [21]

$$
L_{\mathrm{KPZ}}=\xi_{0} e^{8 \pi / g},
$$

and the corresponding time scale, after which scaling behavior according to Eq. (17) sets in, follows from diffusive scaling and is given by [27] $t_{\mathrm{KPZ}}=L_{\mathrm{KPZ}}^{2} / \bar{D}$.
[67] In Eq. (18), $\xi_{0}=\hbar / \sqrt{2 m_{\mathrm{LP}} g_{X} \sqrt{n_{s} n_{i}}}$ is the healing length of the system [68].

Finally, as we mentioned previously, taking into account the compactness of the phase in the KPZ equation in the WA regime, vortices have been predicted to unbind at a scale $L_{v}$ [27],

$$
L_{v}=\bar{\xi}_{0} e^{2 \bar{D} / \bar{\lambda}},
$$

leading to exponential decay of correlations beyond. [69] Thus, the algebraic or KPZ orders in the WA regime might appear only as a finite-size or transient phenomenon. We refer the reader to Appendix B for a detailed description of the physics of the vortices in the WA regime of the compact KPZ equation.

\section{Strongly anisotropic regime}

In the SA regime, the RG flow equations (13) approach the fixed point at $g=0, \Gamma=-1$, belonging to the (dynamical) $X Y$ universality class. Then, for a zero drift term $\mathbf{B}$, the correlations decay as power laws both in space and time [70-72]:

$$
\begin{aligned}
g^{(1)}(\mathbf{r}, t) & \sim \tilde{r}^{-\alpha} F_{X Y}\left(\tilde{r}^{z^{\prime}} /\left(c_{2} t\right)\right) \\
& \sim \begin{cases}\tilde{r}^{-\alpha} & \text { for } \tilde{r}^{z^{\prime}} \gg c_{2} t \\
t^{-\alpha / z^{\prime}} & \text { for } c_{2} t \gg \tilde{r}^{z^{\prime}},\end{cases}
\end{aligned}
$$

where the parameter $c_{2}$ depends on microscopic parameters. The scaling function $F_{X Y}(w)$ behaves asymptotically as $F_{X Y}(w) \sim A_{1}^{\prime}$ for $w \rightarrow \infty$ and as $F_{X Y}(w) \sim A_{2}^{\prime} w^{\alpha / z^{\prime}}$ for $w \rightarrow 0$, where $A_{1}^{\prime}$ and $A_{2}^{\prime}$ are nonuniversal constants. The exponents are $z^{\prime}=2$ and $\alpha=\kappa(\infty) /(4 \pi)$, with the renormalized scaled noise

$$
\kappa(l)=\Delta(l) / \sqrt{D_{x}(l) D_{y}(l)},
$$

evaluated from the RG flow equations for the aKPZ equation in the limit $l \rightarrow \infty$ [30]. We obtain the correlations in the original frame of reference by reverting the coordinate transformation from the comoving frame in which the drift term in Eq. (9) is absent. Replacing $\mathbf{r} \mapsto \mathbf{r}+\mathbf{B} t$ in Eq. (20) yields

$$
g^{\prime(1)}(\mathbf{r}, t) \sim|\mathbf{r}+\mathbf{B} t|^{-\alpha} F_{X Y}\left(|\mathbf{r}+\mathbf{B} t|^{z^{\prime}} /\left(c_{2} t\right)\right) .
$$

We examine the consequences of a nonvanishing drift term $\mathbf{B}$ on the correlations of the system. In particular, the spatial correlations at equal times behave as

$$
g^{(1)}(\mathbf{r}, 0) \sim \tilde{r}^{-\alpha},
$$

which coincides with the result for the case of a vanishing drift term. However, as in the KPZ scaling regime, temporal correlations are modified: 


$$
\begin{aligned}
g^{(1)}(0, t) & \sim(B t)^{-\alpha} F_{X Y}\left((B t)^{z^{\prime}} /\left(c_{2} t\right)\right) \\
& \sim \begin{cases}t^{-\left(\alpha / z^{\prime}\right)} & \text { for } t \ll \tau_{c}^{\prime} \\
t^{-\alpha} & \text { for } t \gg \tau_{c}^{\prime} .\end{cases}
\end{aligned}
$$

Thus, as in the WA regime, the system shows two different exponents, depending on the time scale. Initially, the correlator $g^{(1)}(0, t)$ shows algebraic decay with the characteristic $-\alpha / z^{\prime}$ exponent. At longer times, however, the drift term causes the exponent to decrease to $-\alpha$, resulting in a faster decay of temporal correlations. The crossover time at which the transition between the two regimes occurs is at $\tau_{c}^{\prime} \sim\left(B^{z^{\prime}} / c_{2}\right)^{1 /\left(1-z^{\prime}\right)}$.

We should note that the closed 2D bosonic system in thermal equilibrium, in the absence of drive and dissipation, reveals a slightly different behavior. In such a case, the phase fluctuations obey the following equation: $\partial_{t}^{2} \theta=D_{x}^{\prime} \partial_{x}^{2} \theta+D_{y}^{\prime} \partial_{y}^{2} \theta$, where $D_{x}^{\prime}, D_{y}^{\prime}$ are the squares of the $x$ and $y$ components, respectively, of the speed of sound $[21,57]$. Here, the two-point correlation function shows an algebraic order with the same exponent $\alpha$ for both space and time: $g^{(1)}(\mathbf{r}, t) \rightarrow \tilde{r}^{-\alpha}, t^{-\alpha}$, respectively, with $\alpha>0$ [72]. This is a consequence of the linear dispersion of the gapless Bogoliubov excitation in the $k, \omega \rightarrow 0$ limit.

Including the compactness of the phase in the SA regime does not preclude algebraic order and superfluidity. It leads to a well-known BKT [30] transition between a quasiordered and a disordered phase mediated by the binding or unbinding of vortices. We can estimate the phase boundary for algebraic order by considering a simple argument presented in Refs. [21,30]: We assume that vortices only become relevant at scales where $g$ has flowed to nearly 0 , and hence we can use the RG flow equations (13) even though they do not include vortices [73]. In this scenario, the BKT transition is estimated to occur at $\kappa(\infty)=\pi$, where $\kappa(\infty)$ is the renormalized scaled noise (21) in the limit $l \rightarrow \infty$. This condition defines the phase boundary $\kappa_{0}=\kappa_{*}$ between ordered and disordered phases, which reads

$$
\kappa_{*}=-\frac{4 \pi \Gamma_{0}}{\left(1-\Gamma_{0}\right)^{2}},
$$

where we have used the expression of $\kappa(\infty)$ as a function of the bare parameters $\kappa_{0}$ and $\Gamma_{0}$ [30]. Thus, when $\kappa_{0}<\kappa_{*}$, the system shows algebraic order, whereas if $\kappa_{0}>\kappa_{*}$, the algebraic order is destroyed by vortices, resulting in exponential decay of correlations.

According to the above discussion, while incoherently pumped (and thus, at best, weakly anisotropic) 2D polariton (or other photonic) systems are always disordered in the thermodynamic limit of infinite system size, and algebraic order or superfluidity can only be a finite-size effect, the parametrically pumped polaritons are fundamentally different. The pumping process, which can be chosen at any wave vector, can result in a high level of effective anisotropy, which allows us to enter the SA regime, governed by the $X Y$ equilibrium fixed point, thus ensuring algebraic order up to infinite distances in the thermodynamic limit. Such a high level of anisotropy would not be achievable by a crystal-growth engineering aimed at creating different effective masses in perpendicular directions. Moreover, as we show below, the anisotropy can be changed simply by tuning experimental parameters such as the pump power or the detuning between the excitons and photons, allowing us to easily move between different regimes in one experiment.

\section{EXPLORING SCALING REGIMES OF OPO POLARITONS}

In this section, we show that the OPO-polariton system can be driven from the nonequilibrium WA to an equilibrium-like SA regime by simply tuning the strength of the external pump power and the detuning between the cavity photons and excitons. We then consider implications for finite-size systems. In general, polaritons in the OPO regime (or in the incoherently pumped scenario above condensation threshold) are characterized by a high degree of coherence. This is because the system size in experiments, because of intrinsic disorder in the samples limiting the spatial extent of useful regions, is relativity small in comparison to the relevant length scales of the decay of correlations, especially well above threshold where most experiments operate. Indeed, nondecaying spatial coherence, characteristic of Bose Einstein condensate in 3D, was seen in most cases [74,75], and even observation of the algebraic decay appeared challenging $[31,37,76]$. In order to minimize the influence of the finite size, which masks the underlying physics, we need to focus on samples and regimes, in which we have appreciable decay of coherence for the considered system size. In general, this would correspond to what we call bad samples, where the influence of dissipation is substantial but not strong enough to completely wash out any collective effects. In our opinion, the most promising microcavities are those used in the early days of work on polariton condensation, where collective effects were already seen but the polariton lifetime and the Rabi splitting were quite small by current standards. Thus, we first focus on what we call a bad inorganic microcavity [33,53], and in Sec. IVE, we compare this with better quality samples, characterized by longer lifetimes and larger Rabi splitting, used by most groups today, as well as with organic microcavities.

The bad microcavity is characterized by the following set of parameters: $\hbar \Omega_{R}=4.4 \mathrm{meV}, \hbar \gamma_{j}=0.1 \mathrm{meV}$ (corresponding to a lifetime of $6.6 \mathrm{ps}) ; m_{C}=2.5 \times 10^{-5} m_{0}$, $g_{X}=2 \mu \mathrm{eV} \mu \mathrm{m}^{-2}$. We choose the pump wave vector close to the inflection point of the lower polariton dispersion, $k_{p}=1.4$, which corresponds to $1.61 \mu \mathrm{m}^{-1}$ in dimensional 
units for the bad cavity, and $\omega_{p}=\omega_{\mathrm{LP}}\left(k_{p}\right)$, as shown in Fig. 2. At the mean-field level, the system exhibits upper and lower thresholds for the OPO transition at pump powers indicated by $F_{p}^{\text {lo }}$ and $F_{p}^{\text {up }}$, respectively. Numerically solving exactly the analogue of Eq. (7) for the excitonphoton model and the same set of parameters with zero detuning shows that the system undergoes a BKT-type phase transition at a pump power $F_{p}^{\mathrm{BKT}, \mathrm{lo}} \approx 1.014 F_{p}^{\mathrm{lo}}$ and $F_{p}^{\text {BKT,up }} \approx 0.999 F_{p}^{\text {up }}$ [33]. The value of $k_{s}$ is not determined by the three-modes ansatz (7) [77]. However, the stability analysis shown in Fig. 2 suggests a value of $k_{s} \approx 0.11 \mu \mathrm{m}^{-1}$ for intermediate and high values of $F_{p}$, whereas the system chooses bigger values of $k_{s}$ when approaching the lower threshold, i.e., $k_{s} \in\left[0.11 \mu \mathrm{m}^{-1}, 0.7 \mu \mathrm{m}^{-1}\right]$ (region $\mathrm{C}$ in Fig. 2).

\section{A. Infinite system: Crossover between weakly and strongly anisotropic regimes}

We first focus on the region with small $k_{s}$ (A and B in Fig. 2). The analysis of the anisotropy parameter $\Gamma$ for the bad cavity shows that the system falls into the WA regime, i.e., $\Gamma>0$, at all pump powers when the detuning between the photons and the excitons fulfills $-1.07<\delta_{C X}$. The expected scaling behavior of correlations is discussed in Sec. III C 1. In particular, algebraic decay of correlations is ruled out in this regime. However, this picture changes drastically for lower values of the detuning. When $\delta_{C X} \leq-1.07$, we enter the SA regime, which is expected to have long-range properties that are qualitatively similar to an equilibrium system (see Sec. III C 2). For example, algebraic order can be destroyed by the proliferation of vortices as in the equilibrium BKT transition when the level of the effective noise is large, which is the case when the signal density is low. In Fig. 3, we show three characteristic cases of different detuning superimposed on the phase diagram generated by the relation (25) in the $\Gamma_{0}-\kappa_{0}$ phase space: (i) For $\delta_{C X}=-1.07$, the system is always disordered. It crosses from the disordered nonequilibrium (WA) to the disordered equilibrium-like (SA) regime since $\kappa_{0}>\kappa_{*}$ for all values of $F_{p}$. (ii) $\delta_{C X}=-1.075$ is the most interesting case. The system shows reentrant behavior [21]: By increasing the pump power, we move from the disordered WA to the disordered SA regime; then, by increasing the pump power further, we reach the BKT phase transition to the algebraically ordered phase, followed by a second BKT transition close to the OPO upper threshold back to the SA disordered phase. (iii) For $\delta_{C X}=-1.08$, the stable three-mode solutions lie in the SA regime for all pump powers. For smaller values of $F_{p}$, the system is in the algebraically ordered phase, and it undergoes a BKT phase transition to a disordered phase by increasing $F_{p}$. As can be seen in the left panel of Fig. 4, all three different cases show a nearly linear dependence of $\Gamma$ on the intensity $F_{p}^{2}$ of the external drive.

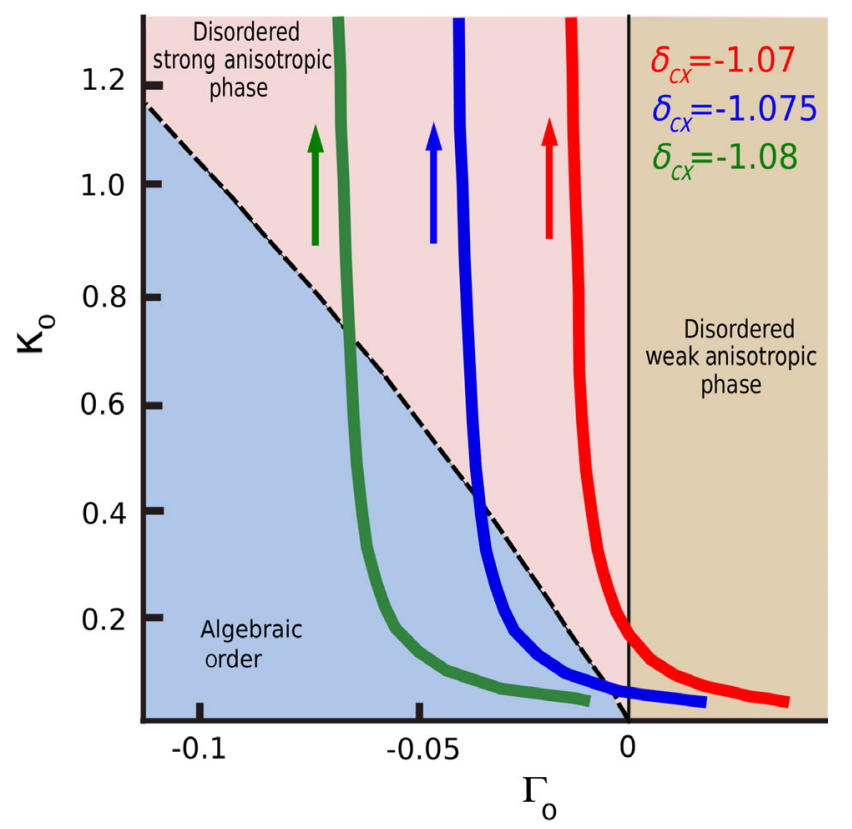

FIG. 3. Crossover between nonequilibrium and equilibriumlike universal regimes. We show stable three-mode OPO configurations in the $\Gamma_{0}-\kappa_{0}$ space for three different detunings, $\delta_{C X}=-1.07$ (green line), -1.075 (blue line), -1.08 (red line), and for $k_{p}=1.4$ and $k_{s}=0.1$ (region A in Fig. 2). The arrows indicate the direction of the increasing external drive strength. The dashed line shows the BKT phase boundary [see Eq. (25)] between algebraically ordered and disordered phases. By increasing the external pump power, for $\delta_{C X}=-1.07$, we cross from the nonequilibrium (WA) to the equilibrium-like (SA) disordered regimes. For $\delta_{C X}=-1.075$, the systems shows reentrance; it starts in the WA, enters the SA algebraically ordered regime, and finally goes back to the disordered but now SA regime. For $\delta_{C X}=-1.08$, we are in the SA equilibriumlike regime for all pump powers, and the system undergoes a BKT transition from an algebraically ordered to a disordered phase by increasing $F_{p}$.

Increasing detuning in the region close to the upper threshold (region A in Fig. 2) is one way to introduce a sufficient level of anisotropy to cross to the equilibriumlike phase. However, there is also another source of anisotropy: The system can be driven to the SA regime by increasing the pump momentum $k_{p}$, leading to an increase of the signal momentum $k_{s}$ by tuning the pump power close to the lower OPO threshold (region $\mathrm{C}$ in Fig. 2). For example, for the bad cavity parameters with $\delta_{C X}=0$ and $k_{p}=2.11 \mu \mathrm{m}^{-1}$ (1.84 in dimensionless units), we enter the SA regime for $k_{s} \approx 0.7 \mu \mathrm{m}^{-1}$, as can be seen in the right panel of Fig. 4.

\section{B. Finite system: Length scales of the weakly anisotropic regime}

In this subsection, based on Eqs. (18) and (19), we estimate the relevant length scales of the WA regime to examine which phases can be seen in current 


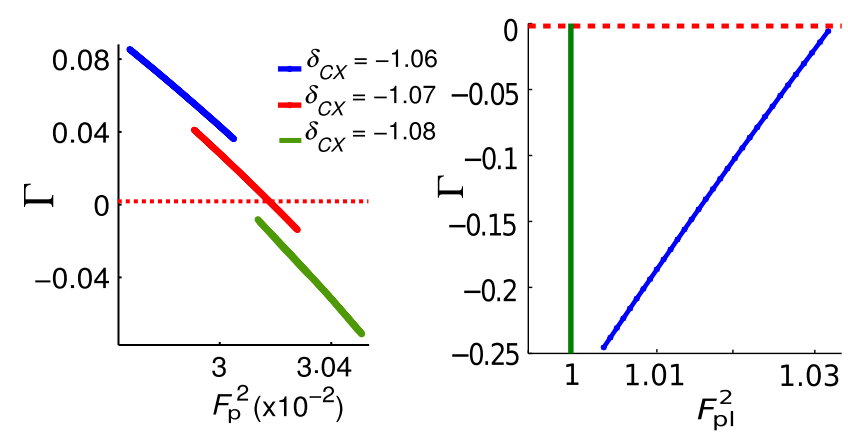

FIG. 4. Changing the effective anisotropy by tuning the driving strength. Left panel: Anisotropy parameter $\Gamma$ as a function of pump power $F_{p}^{2}$ (in the same range as region A in Fig. 2) for different detunings $\delta_{C X}=-1.06$ (blue line), -1.07 (red line), -1.08 (green line) in the stable three-mode OPO regime. Parameters are as in Fig. 3. For $\delta_{C X}=-1.075$, the anisotropy parameter crosses zero (dotted red line). Note that the extent of region A, i.e., the range of pump powers for which the threemode OPO ansatz is stable, depends on the value of the detuning. Right panel: Anisotropy parameter $\Gamma$ as a function of normalized pump power, where $F_{p l} \equiv F_{p} / F_{p}^{\text {lo }}$, at zero detuning but close to the lower OPO threshold, indicated by the green vertical line. The pump and signal have large momenta $k_{p} \approx 1.84$ and $k_{s}=1.0$. The dotted red line marks the $\Gamma=0$ line. This case corresponds to region $\mathrm{C}$ in Fig. 2.

semiconductor microcavities, and whether finite-size effects would hamper the underlying universal physics. We address, in particular, whether the nonequilibrium ordered KPZ phase can ever be seen in semiconductor microcavities. We focus here on our bad cavity parameters as the most promising to explore various phases.

We first explore the WA regime close to the upper threshold (region A in Fig. 2). The parameters of the aKPZ equation for this regime are shown in Fig. 10 in Appendix A. Most of the parameters are approximately constant as a function of the external pump strength, $F_{p}$. The drift term is nonzero only in the direction of the pump wave vector $\mathbf{k}_{p}$, which we have chosen to be along the $x$ axis. The dimensionless nonlinearity $g$ and the noise strength $\Delta$ asymptote to high values, which leads to a small value of $L_{\mathrm{KPZ}}$ [see Eq. (18)], only very close to the upper threshold. As can be seen in Fig. 5, even for the most promising bad cavity parameters, $L_{\mathrm{KPZ}}$ is astronomically large at any reasonable distance from the upper threshold. It goes down to $100 \mu \mathrm{m}$, currently the upper bound for any experiments, only at around 0.999 of the upper threshold (see right panel of Fig. 5). However, this point is already above the BKT transition (green dashed line in Fig. 5), where proliferating vortex-antivortex pairs destroy the KPZ scaling. Additionally, $L_{v} \ll L_{\mathrm{KPZ}}$ for all values of $F_{p}$ apart from those close to the upper threshold already beyond the BKT transition. [Also, note that as explained in Sec. III C 1, close to the upper threshold where fluctuations are strong, the scale at which vortices unbind should be strongly renormalized and smaller than $L_{v}$ in Eq. (19).] Thus, we conclude that in this regime (region A in Fig. 2), KPZ scaling would either be overshadowed by the algebraic order at scales below $L_{v}$ because of the astronomically large length scales required or destroyed by the BKT vortices, resulting in an equilibrium-like behavior in a finite system. The following questions remain: Is there no hope for the KPZ phase in microcavities in two dimensions, and are we only left with equilibrium-like behavior? We address these questions in the next section.

We should also comment that $L_{v}$ drops down to less then $100 \mu \mathrm{m}$ for our bad cavity for some pump powers away from the BKT threshold-a scale that is quite realistic. This suggests that in such a case, free vortices (not of BKT type) should destroy the algebraic order beyond this scale [27] (see Appendix B). However, exact simulations of stochastic dynamics for systems as large as $1000 \mu \mathrm{m}$ [33] do not show any signs of this phase, even at very long times where a steady state has clearly been reached, suggesting suppressed activation by, e.g., an extremely small vortex mobility (Ref. [27] assumed instead a vortex mobility of the order of other scales in the problem), or that attractive interactions between the vortex and antivortex at small distances may, in reality, prevent the free vortices from becoming relevant. It may also be that the rough formula for $L_{v}$ underestimates the real value.

\section{Finite system: Searching for the KPZ phase}

Unlike the incoherently pumped case, our OPO system offers more possibilities for parameter tuning. Interestingly, as we can see in Fig. 6, the system shows a regime at intermediate pump powers, $3.1<\left(F_{p} / F_{p}^{\text {lo }}\right)^{2}<3.5$, where $g$ becomes large and so $L_{\mathrm{KPZ}}$ is small (region B in Fig. 2). In fact, in some parts of this range, $g>1$, meaning that the $\mathrm{KPZ}$ phase is expected at all length scales beyond the healing length. Such a regime does not exist for incoherently driven microcavities. Its presence in the OPO configuration is due to the nonmonotonic behavior of KPZ parameters as a function of pump power, associated with underlying instabilities towards more complex spatial patterns in the system, such as the satellite formation or ring OPOs [77]. However, we also find that $L_{v} \approx \xi_{0}$ (see right panel of Fig. 6); therefore, in principle, the vortex phase could destroy the $\mathrm{KPZ}$ physics. But, because of the suppressed activation observed in numerical studies, the free vortices may never appear. Testing the possibility of the KPZ phase in the middle of the OPO region using the exact stochastic dynamics, and hopefully experiments, would give the final word on this. Note that the only experiment measuring spatial coherence in the OPO configuration focuses on a different regime of powers [75]. Our estimates show that using pump powers a few times the OPO threshold in good quality samples, as far as spatial disorder is concerned, but with relatively short polariton lifetime, is the most promising regime to observe signatures of the KPZ physics. 

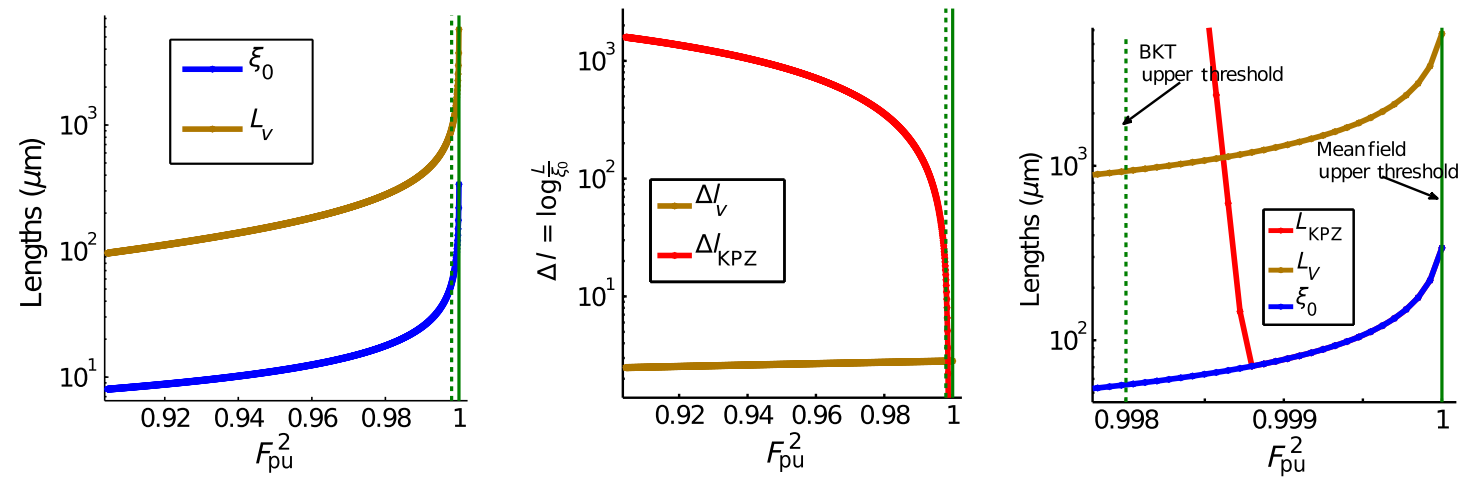

FIG. 5. Characteristic length scales in the WA regime. Left and right panels: Healing length $\xi_{0}$ (blue line), length scale for the vortexdominated disordered phase $L_{v}$ (brown line), and the KPZ length scale $L_{\mathrm{KPZ}}$ (red line) as a function of the pump strength (normalized to the upper threshold, $F_{p u} \equiv F_{p} / F_{p}^{\mathrm{up}}$ ). The vertical dotted (green) line indicates the BKT transition where V-AV pairs proliferate (taken from Ref. [33]), and the vertical solid (green) line shows the mean-field OPO threshold. We use the same parameters as those discussed in the text for the bad microcavity at zero detuning $\delta_{C X}=0, k_{p}=1.4$, and $k_{s}=0.1$. The two different panels correspond to two different ranges in pump strength. Central panel: Logarithmic RG scale for the vortex-dominated phase $\left(\Delta l_{v}\right)$ and for the KPZ phase $\left(\Delta l_{\mathrm{KPZ}}\right)$ as a function of pump strength normalized to the pump at the upper threshold (vertical solid green line). The vertical dotted green line indicates the BKT transition. Note that $L_{v}$ is expected to be strongly renormalized close to the threshold as explained in Appendix B.

\section{Finite system: Crossover between weakly and strongly anisotropic regimes}

In Sec. IV A, we have shown that the infinite polariton system can be tuned between two different universality classes (nonequilibrium KPZ and equilibrium $X Y$ ), with completely different large-scale behaviors, by changing the exciton-photon detuning, and the properties of the drive such as the pump power, $F_{p}$, and wave vector $k_{p}$-easily
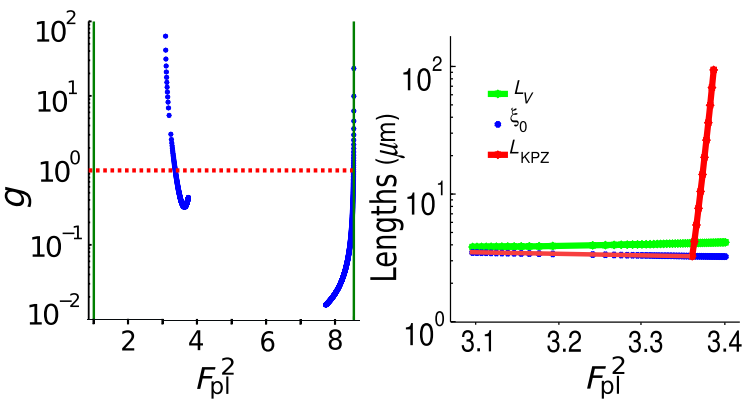

FIG. 6. Characteristic length scales in the WA regime at intermediate pump powers. Left panel: Nonlinear parameter $g$ as a function of the pump strength normalized to the pump at the lower threshold for a stable three-mode OPO solution with zero detuning. The vertical green lines indicate the lower and upper mean-field thresholds, and the red dotted line marks the $g=1$ border. These are the stable solutions at intermediate values of $F_{p}$ (region B in Fig. 2). Right panel: Characteristic length scales at the intermediate $F_{p}$ shown in the left panel. Healing length $\xi_{0}$ (blue line), length scale for vortex-dominated disordered phase $L_{v}$ (green line), and the KPZ length scale $L_{\mathrm{KPZ}}$ (red line) as a function of pump strength normalized to the pump at the upper threshold. Note that to the left of the nearly vertical increase of $L_{\mathrm{KPZ}}$, its value is indistinguishable from the healing length. realizable in current experiments. Here, we consider how this crossover is affected by the finite size.

We consider the case with detuning $\delta_{C X}=-1.07$ close to the upper OPO threshold (region A in Fig. 2). As we discussed, for this detuning, the system can move from the nonequilibrium (WA) to an equilibrium-like (SA) regime by increasing the external pump power. If the system is infinite, for these parameters, it is in the disordered phase, characterized by the exponential decay of correlations, in both regimes. However, the system may show algebraic order up to a certain length scale $L_{\mathrm{BKT}}$ in the SA regime or $L_{v}, L_{\mathrm{KPZ}}$ in the WA regime. In the first case, this would require $\kappa_{*}<\kappa(l)<\pi$ [see Eq. (25)], and $L_{\mathrm{BKT}} \equiv \xi_{0} e^{l_{\mathrm{BKT}}}$ is obtained by considering the "BKT-transition criterion": $\kappa\left(l_{\mathrm{BKT}}\right) \sim \pi$. We calculate $L_{\mathrm{BKT}}$ following Ref. [30] and the $\mathrm{RG}$ flow equations (13), and the results are displayed in Fig. 7. We obtain that $L_{\mathrm{BKT}}$ takes reasonable physical values for $\Gamma \approx-0.0145$. When $\Gamma>-0.0145, L_{\mathrm{BKT}} \rightarrow \infty$, which indicates that the algebraic order appears at all realistic physical length scales. However, when $\Gamma<-0.0145, L_{\mathrm{BKT}} \sim \xi_{0}$; thus, the system is in a disordered phase at all length scales beyond the healing length.

Considering this behavior, we find that there are two interesting scenarios at intermediate length scales when driving the system from the WA to the SA regime, as indicated by double arrows $A$ and $B$ in Fig. 7. The first case, arrow $A$, shows a transition between the disordered phase in the nonequilibrium WA regime to the algebraically ordered phase in the equilibrium-like SA regime by increasing the pump power and, consequently, crossing $\Gamma=0$. This phenomenon appears for $L$ such that $L_{v}<L<L_{\mathrm{BKT}}$. In the second scenario (arrow $B$ in Fig. 7), the system can be driven from the WA to the SA regime without changing the 


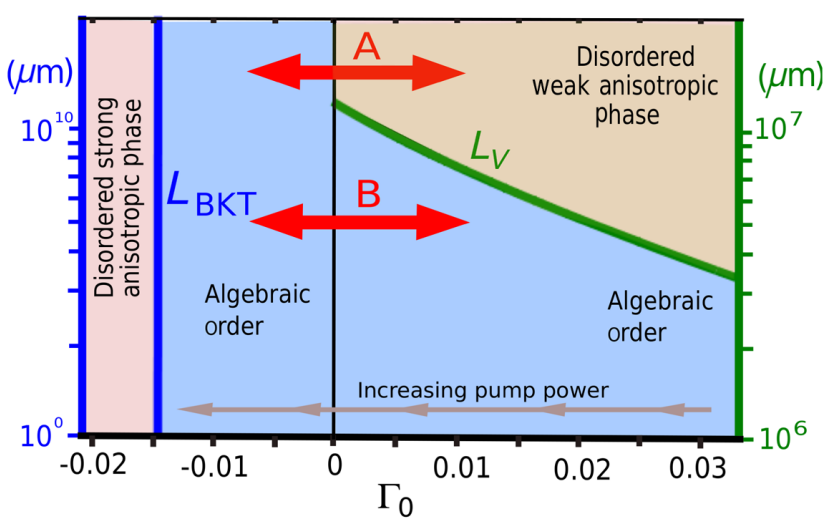

FIG. 7. Crossover between SA (negative $\Gamma_{0}$ ) and WA (positive $\left.\Gamma_{0}\right)$ regimes at finite length scales. Length scale for the vortexdominated phase in the WA regime $L_{v}$ (green line) and the disordered phase in the SA $L_{\mathrm{BKT}}$ (blue line) as a function of the anisotropy parameter, tunable by the drive, for the polariton system with $\delta_{C X}=-1.07$. The double red arrow A indicates a transition from the disordered WA to an algebraically ordered SA phase by increasing the external pump power in a system of size $L$ such that $L_{v}<L<L_{\mathrm{BKT}}$. The double red arrow B indicates a transition from the ordered WA regime to the ordered SA regime by increasing the external pump power in a system of size $L$, where $L<L_{v}<L_{\mathrm{BKT}}$.

phase, i.e., maintaining the algebraic order in both cases. This situation occurs for $L$ fulfilling $L<L_{v}, L_{\mathrm{BKT}}$. Scenario $A$ requires length scales of the order of meters for our bad cavity parameters. Thus, scenario $B$ is more likely in current experiments. Realizing scenario A would require increasing the dissipation in a controlled way so that not all the collective effects are washed out and the OPO survives.

Finally, we can ask whether a transition between WA $(\Gamma>0)$ and SA $(\Gamma<0)$ regimes is possible in conditions of strong KPZ nonlinearity $(g>1)$ where KPZ scaling would show at all length scales beyond the healing length. This would mean crossing a phase with stretched exponential decay of correlations to a phase with algebraic order as in equilibrium systems below the BKT transition. We find that such KPZ-to-algebraic-order crossover as a function of pump power is indeed possible at finite detuning (see Fig. 8 for the bad cavity system).

\section{E. Different experimental systems}

In the previous section, we studied in detail the bad cavity configuration characterized by a relatively large photon decay rate with a polariton lifetime of $\tau \approx 6.6 \mathrm{ps}$, as the most promising for observation of different phases. However, current state-of-the-art inorganic microcavities are characterized by much longer polariton lifetimes. Indeed, typical inorganic cavities have polariton lifetimes of $\tau \approx 30$ ps [78], whereas the best cavities show polariton lifetimes $\tau \approx 150$ ps [31,79]. We refer to the latter as good cavities. Most inorganic samples are characterized by Rabi splittings $\Omega_{R}$ and exciton-exciton interaction strengths $g_{X}$
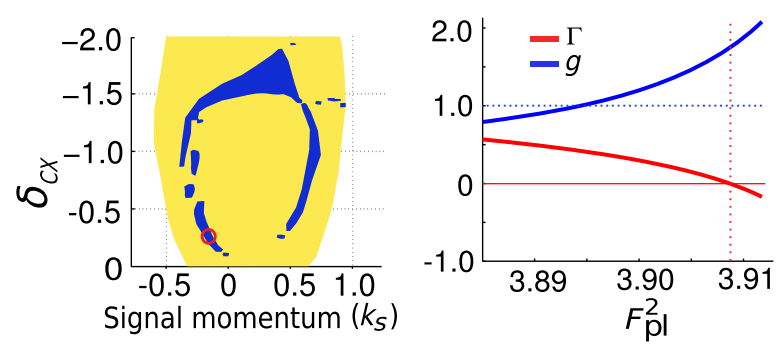

FIG. 8. Crossover between the KPZ scaling and the SA (negative $\Gamma_{0}$ ) regime with algebraic order. Left panel: Regions of detuning between the cavity photons and the excitons $\delta_{C X}$ and signal momentum $k_{s}$ (dark blue) for which the system can exhibit a crossover between the KPZ phase and algebraically ordered phase by tuning the pump strength $F_{p}$. The yellow region marks stable KPZ solutions. Right panel: $g$ and $\Gamma$ as a function of the normalized pump strength $F_{\mathrm{pl}}^{2}$ for $k_{s}=-0.15$ and $\delta_{C X}=-0.25$ (small red circle in the left panel). The crossover between the $\mathrm{KPZ}$ and the SA regimes occurs at $F_{\mathrm{pl}}^{2} \simeq 3.909$ : $\Gamma$ changes sign (vertical red dotted line) while $g>1$ (dotted blue horizontal line). Here, $k_{p}=1.4$, and we consider all values of $k_{s}$ for which there is a nonzero mean-field solution and a stable aKPZ equation. Note that the stability analysis indicates that in the blue regions, the three-mode ansatz is unstable. This is confirmed by numerical studies [77], which show that the steady-state polariton field can develop several secondary modes in addition to the three main ones. However, the secondary modes are typically orders of magnitude weaker, and we expect them to give only minor quantitative corrections to the values of $g$ and $\Gamma$ we find here.

comparable to the ones used in the previous section for the

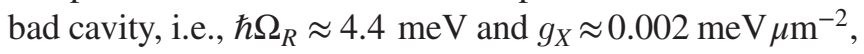
respectively. On the other hand, organic microcavities have extremely low photon lifetimes but high Rabi splittings and relatively small exciton-exciton interaction strength. Typical values for organic cavities are $\tau \approx 5.5 \times 10^{-2} \mathrm{ps,}$ $\hbar \Omega_{R} \approx 1000 \mathrm{meV}$ and $g_{X} \approx 10^{-6} \mathrm{meV}_{\mu} \mathrm{m}^{-2}$ [80].

In this section, we present a comparison of the relevant length scales for these different cavities, i.e., the bad, typical, good, and organic cavities with zero detuning between the cavity photons and the excitons. We first focus on the OPO region at high pump powers (region A in Fig. 2). The results are listed in Table I. The first four rows show the length scale for the vortex-dominated phase $\left(L_{v}\right)$, the KPZ phase $\left(L_{\mathrm{KPZ}}\right)$, the healing length $\left(\xi_{0}\right)$, and the corresponding normalized pump power $F_{n p}^{2}$ at a point where the $L_{v}$ is smallest within stable three-mode OPO solutions (see left panel in Fig. 5 as example for the bad cavity). The estimate for $L_{v}$ is done using $\xi_{0} \approx \bar{\xi}_{0}$ [cf. the discussion around Eq. (19)], which may not be realistic, so this information has to be taken with a grain of salt. In addition, we observe an $L_{v}$ that takes reasonable physical values for the bad cavity and organic samples, whereas the $\mathrm{KPZ}$ scale $L_{\mathrm{KPZ}}$ is unreachable.

As we mentioned in the last section, close to the upper threshold, the KPZ length scale drops down significantly, 
TABLE I. Comparison of different experimental microcavities. Each column contains details for one of the four different considered cavities: bad, typical, good, and organic samples for $k_{p}=1.4$ and $k_{s}=0.1$. The first four rows indicate characteristic length scales for the vortex-dominated phase $\left(L_{v}\right)$, for the KPZ phase $\left(L_{\mathrm{KPZ}}\right)$, the healing length $\left(\xi_{0}\right)$, and normalized pump power $\left(F_{p u}^{2}\right)$ at the point where $L_{v}$ reaches the minimum value in the stable three-mode OPO region close to the upper threshold (region A in Fig. 2). The fifth, sixth, and seventh rows show $L_{\mathrm{KPZ}}$, $\xi_{0}$, and $F_{p u}^{2}$, where $L_{\mathrm{KPZ}} \approx L_{v}$, close to the upper threshold. The blank spaces for the good and the organic samples mean that this crossing point occurs practically at the upper threshold. The last row shows the values of the drift term $B_{d}$ in dimensional units, which is approximately constant for the different values of the external drive (see Fig. 10 for the bad cavity case). It is clear that the KPZ length scale is unrealistic in regions $\mathrm{A}$ and $\mathrm{C}$ of the phase diagram presented in Fig. 2, leaving region B as the only potentially promising regime.

\begin{tabular}{lcccc}
\hline \hline Name & Bad & Typical & Good & Organic \\
\hline$L_{v}(\mu \mathrm{m})$ & $10^{2}$ & $10^{8}$ & $10^{27}$ & $2 \times 10^{2}$ \\
$L_{\mathrm{KPZ}}(\mu \mathrm{m})$ & $10^{10^{3}}$ & $10^{10^{4}}$ & $10^{10^{5}}$ & $10^{10^{8}}$ \\
$\xi_{0}(\mu \mathrm{m})$ & 10 & 10 & 10 & 1 \\
$F_{n p}^{2}$ & 0.9 & 0.84 & 0.97 & 0.84 \\
$L_{\mathrm{KPZ}}(\mu \mathrm{m})$ & $10^{3}$ & $10^{10}$ & & \\
$\xi_{0}(\mu \mathrm{m})$ & 60 & $10^{2}$ & & \\
$F_{p u}^{2}$ & 0.9988 & 0.9999 & 1.0000 & 1.000 \\
$B_{d}(\mu \mathrm{m} / \mathrm{ps})$ & 0.30 & 0.18 & 0.30 & 2.69 \\
\hline \hline
\end{tabular}

reaching $\xi_{0}$ and $L_{v}$. (See right panel in Fig. 5 for an example of the bad cavity. Also, note that $L_{v}$ is expected to be strongly renormalized close to the threshold as explained in Appendix B.) The fifth, sixth, and seventh rows show the values of $L_{\mathrm{KPZ}}, \xi_{0}$, and $F_{l u}^{2}$ at this point. We find that only for the bad and typical configurations can this intersection point be distinguished from the upper threshold. The last row shows the magnitude of the drift term of the aKPZ equation in dimensionless units for $k_{p}=1.4$. The organic cavity has the highest values, whereas the typical configuration has the lowest one. We can conclude that in the stateof-the-art microcavities near the OPO threshold, the length scales associated with the KPZ or vortex-dominated phases are absolutely unrealistic, and the physics is dominated by the equilibrium-like BKT transition between disorder and algebraically ordered phases.

Finally, we examine whether the strong KPZ nonlinearity, which would result in stretched exponential decay of correlations at all length scales beyond the healing length (cf. Sec. IV C), is also present in other cavity configurations. We find that for longer-lifetime cavities, this regime moves to higher pump powers above the OPO threshold with respect to the bad cavity configuration (see Fig. 9). However, for all cavities other than the bad cavity, this regime falls into a region where the three-mode ansatz is unstable towards more complex solutions. Examining

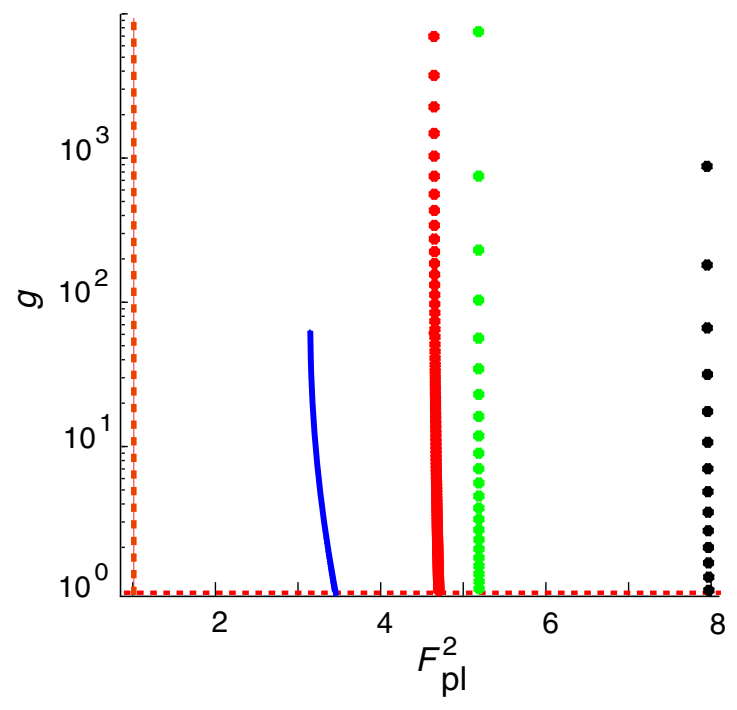

FIG. 9. Regions potentially exhibiting a KPZ phase for different experimental microcavities. The KPZ nonlinearity parameter $g$ (in a region where $g>1$ ) is shown as a function of the normalized pump strength $F_{p l}^{2}$ for four different microcavities: bad (blue line), typical (red dots), good (black dots), and organic (green dots) cavities. We present the regions that could potentially show KPZ scaling at all length scales beyond the healing length, i.e., $g>1$ and $\Gamma>1$ (see Sec. IV A). The dashed horizontal line marks the $g=1$ border, whereas the dashed vertical line indicates the normalized lower mean-field threshold, i.e., $F_{p l}^{2}=1$. Note that only for the bad cavity is the three-mode ansatz stable in the region shown. In this plot, we consider $k_{p}=1.4$ and $k_{s}=0.1$ for the bad and good cavities and $k_{p}=1.6$ and $k_{s}=0.06$ for the typical and organic cavities.

whether the KPZ scaling persists beyond the three-mode ansatz is beyond the scope of this work.

\section{F. Summary}

We explored the wealth of scaling regimes accessible with coherently driven microcavity polaritons. The basis of our analysis is a long-wavelength effective description of OPO polaritons in terms of the compact anisotropic KPZ equation (9). A key point is that while the dynamics of both incoherently and coherently driven polaritons can be mapped to the aKPZ equation, in the latter case, a much wider range of parameters is accessible by tuning the pump strength, the exciton-photon detuning, and the pump wave vector. Ultimately, the reason for this high versatility of OPO polaritons is the different physics formally leading to the same long-wavelength description. In particular, the strongly fluctuating Goldstone mode is the phase of the condensate in the case of incoherent pumping, while it is the relative phase of signal and idler modes for polaritons in the OPO regime. The crucial merit of this high tunability is that the rich scaling behavior of the compact anisotropic KPZ equation becomes accessible in a single experimental platform. 
Experimentally, the scaling regimes can be distinguished by measuring the spatial and temporal decay of the firstorder coherence function (4): In the WA regime, strongly nonlinear fluctuations of the phase lead to stretched exponential decay of correlations, with exponents in the spatial and temporal "directions" given by the KPZ roughness and dynamical exponents. However, the nonlinearity also leads to screening of the interactions of vortices, which could result in their unbinding and thus preclude the observation of KPZ scaling as discussed in Appendix B. Either way, both effects are beyond the physics of 2D superfluids in equilibrium, and it would be intriguing to see them in experiments. They occur beyond length and time scales that are exponentially large in the KPZ nonlinearity. Thus, their observation is greatly facilitated in OPO polaritons, in which this nonlinearity can become of order one. As the OPO threshold is approached in the WA regime, order on shorter scales is destroyed through the usual KT mechanism of vortex proliferation.

In the SA regime, the nonlinearity is irrelevant, and the effective long-wavelength theory becomes linear as in thermal equilibrium. Thus, true algebraic order is possible. [81] The effective renormalized noise level that determines whether the system is in the ordered or disordered phase depends, in a nontrivial way, on the pumping strength. In particular, it shows reentrant behavior: Upon increasing the driving strength, the system can first enter the ordered phase and then leave it again.

The most intriguing prospect is thus to cross from the WA to the SA regime simply by tuning the pumping strength. Order is then established because of a change in the effective degree of anisotropy. This is shown to be, in principle, possible close to the upper OPO threshold for negative detuning (i.e., blue curve in Fig. 3). Then, as the pumping strength is increased, the system crosses from WA to SA and, within the SA regime, from disordered to ordered and back. Another possibility to enter the SA regime close to the lower OPO threshold is to increase the pump momentum. This shows that all universal scaling regimes of the compact anisotropic KPZ equation are, in principle, accessible with OPO polaritons-in a sufficiently large system.

The relevant length scales above which asymptotic universal behavior can be observed are determined by the strength of the nonlinearity in the KPZ equation-a stronger nonlinearity implies shorter crossover scales and is thus favorable for experimental observation. The mere presence of nonlinearity in turn reflects that the system is fundamentally out of thermal equilibrium, and its value is enhanced if the dynamics is dominated by drive and dissipation; i.e., when polaritons have short lifetimes, strong pumping is required. Our estimates for the relevant scales to enter universal scaling regimes thus focus on a bad cavity (values of parameters are given at the beginning of Sec. IV). We find that while close to the OPO threshold both $L_{\mathrm{KPZ}}$ and $L_{v}$, beyond which we expect KPZ scaling and unbinding of vortices (induced by the screening of interactions due to the KPZ nonlinearity), respectively, are beyond realistic system sizes, there is a very promising regime away from the lower and upper thresholds in which nonlinear effects are strong and $L_{\mathrm{KPZ}}$ and $L_{v}$ are of the order of the condensate healing length. It would be desirable to explore this regime experimentally or by solving the stochastic equations of motion (7) numerically.

Finally, we discuss the crossover from WA to SA in finite-size systems while approaching the upper threshold. The most interesting scenario would be to make a transition from a disordered to an algebraically ordered phase (up to the size of the system) by tuning the effective anisotropy from weak to strong (arrow A in Fig. 7). However, implementing this with the bad cavity parameters is not feasible in realistic system sizes. On the other hand, by optimizing the exciton-photon detuning and moving to higher pump powers (around 4 times the lower OPO threshold in Fig. 6), we can cross from the WA to the $\mathrm{SA}$ regime in conditions where the dimensionless KPZ nonlinearity is larger than one implying KPZ scaling at all length scales beyond the healing length. From the experimental point of view, this means crossing from a KPZ phase with stretched exponential decay of spatial and temporal coherence to an algebraically ordered phase by increasing the pump strength in a controlled way.

\section{CONCLUSIONS}

This work provides a first detailed theoretical study of macroscopic nonequilibrium effects independent of the microscopic parameters of realistic experimental systems in two dimensions-driven microcavity polaritons in the OPO configuration. We establish that these systems can act as a natural and unifying laboratory for the exploration of a variety of intrinsic nonequilibrium phenomena in experiments, including the so-far experimentally elusive KPZ phase in two dimensions. Key features of such systems are the high tunability of microscopic parameters, in general, and of the spatial anisotropy of the microscopic physics, in particular. This unique situation impacts strongly on the ensuing macroscopic physics and allows one to move between different phases with different universal properties. In particular, we find that in the low-pump-power regime of the OPO configuration, the long-distance physics is governed by the nonequilibrium fixed point of the KPZ universality class. In the middle region of the OPO phase diagram, the crossover length scale to KPZ physics becomes small, approaching the order of the healing length and suggesting that the KPZ order in a quantum system might indeed be observed in experiments on semiconductor microcavities - in stark contrast to incoherently pumped polaritons. This provides a promising new direction for experiments in the OPO configuration, as well as an exciting prospect for the observation of KPZ physics in 
actual physical platforms, which so far has been elusive. On the other hand, in the same OPO configuration but at higher pump powers, we demonstrate that despite their intrinsic driven-dissipative nature and highly nonthermal occupations, such systems can be driven to a phase characterized by the dynamical $X Y$ universality class. They then show emergent equilibrium behavior at asymptotic length scales, with algebraic order and superfluidity even in the thermodynamic limit. This effect is rooted in the strong anisotropy that is feasible in the OPO configuration, again unaccessible with incoherently driven polaritons, where algebraic order and superfluidity can only be finite-size effects that are absent in the thermodynamic limit. Our findings shine a new light on the ongoing debate about the nature of the polariton ordered phase in semiconductor microcavities, and motivate various directions of theoretical and experimental research. In particular, they suggest more elaborate and targeted numerical scrutinization of promising parameter regimes in the search for KPZ physics. They also call for pushing the understanding of vortices in the compact KPZ equation in two dimensions, which seems to be trapped in an extremely long-lived metastable plateau. They also define a new theoretical challenge on the competition of nonequilibrium physics and anisotropy in conjunction with vortices. Finally and crucially, they may also fuel the search for KPZ physics in other collective light-matter platforms. Possible platforms are ultracold atomic gases in an atom laser configuration [82] or arrays of plasmonic wave guides coupled to atoms by proximity effects [83,84].

\section{ACKNOWLEDGMENTS}

We would like to thank E. Altman and L. He for helpful discussions. We acknowledge support from EPSRC (Grants No. EP/I028900/2 and No. EP/K003623/2). L. S. acknowledges funding from the ERC Synergy Grant UQUAM. S. D. acknowledges funding by the German Research Foundation (DFG) through the Institutional Strategy of the University of Cologne within the German Excellence Initiative (ZUK 81), and by the European Research Council via ERC Grant Agreement No. 647434 (DOQS). This research was supported in part by the National Science Foundation under Grant No. NSF PHY11-25915.

\section{APPENDIX A: MAPPING TO THE KPZ EQUATION AND NUMERICAL RESULTS}

In this section, we consider in detail the mapping in the long-range limit between the stochastic equations (7), describing the dynamics of the OPO condensate, and the aKPZ equation (9). First, as mentioned in Sec. III B, we introduce the hydrodynamic phase-amplitude representation (8) into the dynamical stochastic equations (7). In particular, considering the $U(1)$ symmetry of the system [see Eq. (1)], it is convenient to change from the phase variables $\left\{\theta_{s}, \theta_{i}\right\}$ to $\left\{\theta_{+}, \theta\right\}$ :

$$
\begin{aligned}
& \theta_{s}=\theta+\theta_{+}, \quad \theta=\frac{1}{2}\left(\theta_{s}-\theta_{i}\right), \\
& \theta_{i}=-\theta+\theta_{+}, \quad \theta_{+}=\frac{1}{2}\left(\theta_{s}+\theta_{i}\right),
\end{aligned}
$$

where $\theta$ indicates the phase difference between the signal and the idler states. Thus, we get the following set of coupled dynamical equations for the fluctuations:

$$
\begin{aligned}
& \partial_{t} \theta+D_{s}(\theta)+\partial_{t}\left(\theta_{+}-i \pi_{s} / \sqrt{\rho_{s}}\right)=M_{s} \phi-\tilde{\xi}_{s}, \\
& \partial_{t} \theta+D_{i}(\theta)+\partial_{t}\left(-\theta_{+}+i \pi_{i} / \sqrt{\rho_{i}}\right)=M_{i} \phi+\tilde{\xi}_{i}, \\
& \partial_{t}\left(\theta_{p}-i \pi_{p} / \sqrt{\rho_{p}}\right)=M_{p} \phi-\tilde{\xi}_{p},
\end{aligned}
$$

where $\tilde{\xi}_{j}=\xi_{j} /\left(\sqrt{\rho_{j}} e^{i \varphi_{j}}\right)$, and we have introduced the phase variables $\theta, \theta_{+}$in place of $\theta_{s}, \theta_{i}$ according to Eq. (A1). The phase $\theta$ is kept to all orders, whereas the fluctuations grouped together under $\phi^{T}=\left(\pi_{s} \pi_{i} \pi_{p} \theta_{+} \theta_{p}\right)$ are considered up to linear order. Note that $M_{j}$ is the mass matrix of the mode $j$, and its coefficients depend on the mean-field parameters of the system. The operators $D_{s}(\theta)=-i \omega_{1 s} \nabla \theta+i \nabla^{T} \omega_{2 s} \nabla \theta+\nabla^{T} \theta \omega_{2 s} \nabla \theta$ and $D_{i}(\theta)=$ $-i \omega_{1 i} \nabla \theta+i \nabla^{T} \omega_{2 i} \nabla \theta-\nabla^{T} \theta \omega_{2 i} \nabla \theta$ are obtained by considering the expansion of the polariton dispersion relation around $\mathbf{k}_{s}$ and $\mathbf{k}_{i}$ [see Eq. (10)].

Because of the U(1) symmetry of the system, Eq. (1), the phase fluctuation $\theta$ is a gapless mode (there is no "mass" term for such a fluctuation), whereas the fluctuations grouped under $\phi$ are all gapped. Note that we neglect the following terms containing temporal and spatial derivatives: $\pi_{j} \partial_{t} \theta_{j}, \nabla \pi_{j}, \quad \nabla^{T} \omega_{2 j} \nabla \pi_{j}, \quad \pi_{j} \nabla \theta_{j}, \quad \pi_{j} \nabla^{T} \omega_{2 j} \nabla \theta_{j}$, $\pi_{j} \nabla \theta_{j}$, and $\pi_{j} \nabla^{T} \theta_{j} \omega_{2 j} \nabla \theta_{j}$, which are small compared to the masslike contributions in the long-range limit of $\pi_{j}$. Applying the same criterion, we also omit the spatial derivatives of $\theta_{+}$and $\theta_{p}$. The next step is to consider the real and imaginary parts of Eq. (A2) and neglect the time derivatives of the massive modes since they are "slow" variables [21]:

$$
\left(\begin{array}{c}
\partial_{t} \theta+\operatorname{Re}\left[D_{s}(\theta)+\tilde{\xi}_{s}\right] \\
\operatorname{Im}\left[D_{s}(\theta)+\tilde{\xi}_{s}\right] \\
\partial_{t} \theta+\operatorname{Re}\left[D_{i}(\theta)-\tilde{\xi}_{i}\right] \\
\operatorname{Im}\left[D_{i}(\theta)-\tilde{\xi}_{i}\right] \\
\operatorname{Re}\left[\tilde{\xi}_{i}\right] \\
\operatorname{Im}\left[\tilde{\xi}_{i}\right]
\end{array}\right)=\left(\begin{array}{c}
\operatorname{Re}\left[M_{s}\right] \\
\operatorname{Im}\left[M_{s}\right] \\
\operatorname{Re}\left[M_{i}\right] \\
\operatorname{Im}\left[M_{i}\right] \\
\operatorname{Re}\left[M_{p}\right] \\
\operatorname{Im}\left[M_{p}\right]
\end{array}\right) \phi .
$$

In Eq. (A3), the massive fluctuations can be eliminated; i.e., they can be expressed solely in terms of the spatial and time derivatives of $\theta$, and the noise terms. By considering the last five relations of Eq. (A3), we can express the gapped modes as 


$$
\phi^{T}=\phi^{T}\left(\partial_{t} \theta, \partial_{x} \theta, \partial_{y} \theta, \partial_{x}^{2} \theta, \partial_{y}^{2} \theta, \tilde{\xi}_{s}, \tilde{\xi}_{i}, \tilde{\xi}_{p}\right)
$$

and substitute into the first equation of (A3) to obtain a single dynamical stochastic equation for the gapless $\theta$ variable, which has the form of the KPZ equation with a drift term [Eq. (9)]. The different KPZ coefficients in Eq. (9) read

$$
\begin{aligned}
D_{m} & =\alpha_{1} \omega_{2 s, m m}+\alpha_{2} \omega_{2 i, m m}, \\
\lambda_{m} & =\alpha_{3} \omega_{2 s, m m}+\alpha_{4} \omega_{2 i, m m}, \\
B_{m} & =\alpha_{5} \omega_{1 s, m}+\alpha_{6} \omega_{1 i, m}, \\
2 \Delta & =\left(\beta_{1}^{2}+\beta_{2}^{2}\right) \gamma_{s}+\left(\beta_{3}^{2}+\beta_{4}^{2}\right) \gamma_{i}+\left(\beta_{5}^{2}+\beta_{6}^{2}\right) \gamma_{p}
\end{aligned}
$$

for $m=x, y$. The $\alpha$ and $\beta$ coefficients come from Eq. (A4), while the $\omega$ coefficients originate from the lower polariton dispersion (10): $\omega_{1 j}^{T}=\left(\omega_{1 j, x}, \omega_{1 j, y}\right)$ and

$$
\omega_{2 j}=\left(\begin{array}{cc}
\omega_{2 j, x x} & 0 \\
0 & \omega_{2 j, y y}
\end{array}\right) .
$$

As an example, in Fig. 10, we show the numerical values of the different KPZ coefficients as a function of pump power close to the OPO upper threshold and at zero detuning for the set of parameters given in Sec. IV characterizing what we call a bad cavity. The system is WA in the region shown since $\lambda$ 's have the same sign. The drift term acts in the $x$ direction because of the choice of the pumping wave vector, i.e., $\mathbf{k}_{p}=\left(k_{p}, 0\right)$. In this WA regime, the KPZ coefficients do not vary excessively with respect to the external pump power apart from $g$ and $\Delta$, which asymptote to infinity at the upper mean-field OPO threshold.
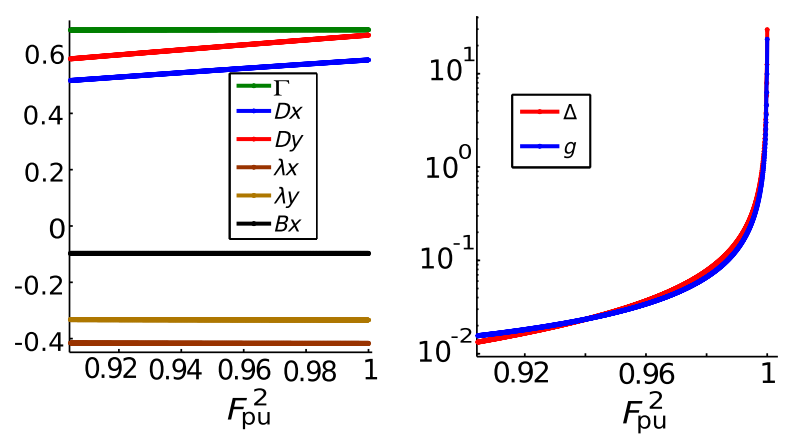

FIG. 10. The aKPZ coefficients for the bad cavity configuration with zero detuning. Left panel: Diffusion coefficients $\left(D_{x}, D_{y}\right)$, nonlinearities $\left(\lambda_{x}, \lambda_{y}\right)$, anisotropy parameter $(\Gamma)$, and drift term $B_{x}$ as a function of the pump power normalized to the upper threshold. These parameters vary little with the external pump power. Right panel: Dimensionless nonlinearity $g$ and noise parameter $\Delta$ as a function of the normalized pump power. We observe the asymptotic growth of these parameters close to the upper OPO threshold.

\section{APPENDIX B: VORTICES IN THE COMPACT KPZ EQUATION IN THE WA REGIME}

In thermal equilibrium, vortices of opposite charge attract each other with a force that falls off as $\sim 1 / r$, like charges in a 2D Coulomb gas. This leads to the formation of closely bound dipoles at low temperatures, whereas at high temperatures the interaction is screened at large distances and the bound state of vortex-antivortex pairs is no longer stable. The fundamental qualitative modification in a driven-dissipative system is that, because of the $\mathrm{KPZ}$ nonlinearity, the vortex interaction is screened even in the absence of noise-induced fluctuations [85] (a situation corresponding to zero temperature in an equilibrium problem). Therefore, even without noise, there is a finite screening length beyond which the interaction is suppressed exponentially. For weak nonlinearities, this length scale is given by expression (19).

As explained above, the screening of the interaction between vortices beyond the scale $L_{v}$ is solely due to the nonlinear terms in the KPZ equation. At finite noise, when fluctuations lead to the creation of vortex-antivortex pairs, there is additional screening induced by the polarization of bound pairs (which leads to unbinding above the critical temperature in the usual equilibrium BKT problem). This effect can only be captured in a proper RG treatment [27] and is not incorporated in the estimate Eq. (19). Associated with $L_{v}$ is a time scale $t_{v}$ for vortices to escape the region of attractive interactions at distances below $L_{v}$. Then, a vortexdominated regime characterized by exponential decay of correlations and in which superfluidity is destroyed should appear above the scales $L_{v}$ and $t_{v}$. If these scales are smaller than the corresponding KPZ scales defined above, then the scaling forms (16) and (17) will be completely masked by the vortex-induced exponential decay. Indeed, for weak KPZ nonlinearities, some of us estimated that $L_{v} \ll L_{\mathrm{KPZ}}$ and $t_{v} \ll t_{\mathrm{KPZ}}$ [27]. The latter estimate for the time scales relies on the assumption that the mobility of vortices is not atypically small, i.e., not much smaller than the diffusion coefficients $D_{x, y}$ in the aKPZ equation (9), which are determined by the same microscopic physics.

Vortex unbinding induced by nonequilibrium conditions has so far remained elusive in experiments with incoherently pumped, and thus isotropic, polariton systems, as well as in the stochastic simulations described in Ref. [33]. This could be ascribed to the limited length and time scales available to experiments and numerics, but it could also be taken as an indication that the time scale $t_{v}$ for vortices to unbind is indeed much larger than expected, thus leaving open the intriguing possibility to observe KPZ scaling if the system is initialized in a vortex-free state and parameters are chosen such that the dimensionless nonlinearity $g$ is large [leading to small values of $t_{\mathrm{KPZ}}$ and $L_{\mathrm{KPZ}}$, see Eq. (18)]. The most promising regime for observing KPZ physics is described in Sec. IV C. 


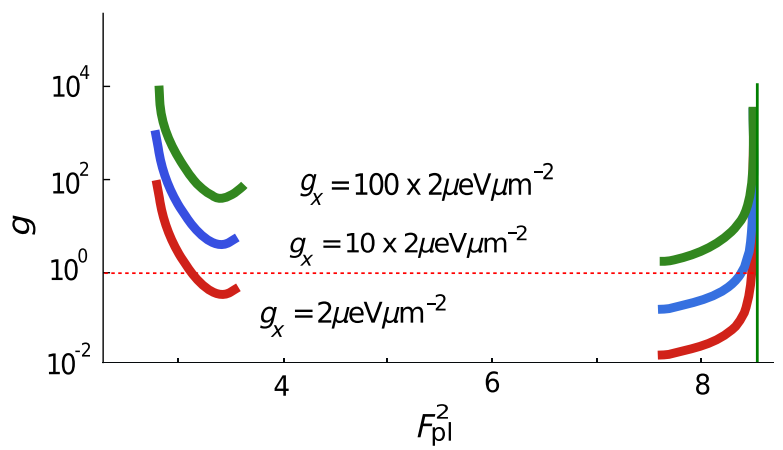

FIG. 11. Dependence of the nonlinear parameter $g$ on excitonexciton interaction strengths. We show $g$ for three different values of $g_{X}$ as a function of the normalized external pump power $F_{p l}$ with the lower threshold for a cavity with zero detuning. We observe that the system shows larger values of $g$ when increasing the exciton-exciton interaction.

\section{APPENDIX C: EFFECTS OF THE EXCITON-EXCITON COUPLING STRENGTH}

Throughout this paper, we considered the exciton-exciton interaction strength to be $g_{X}=2 \mu \mathrm{eV} \mu \mathrm{m}^{-2}$. However, the true value of $g_{X}$ is still the subject of debate, and different values have been reported in the literature (see, for example, Ref. [86]). We consider $g_{X}=2 \mu \mathrm{eV} \mu \mathrm{m}^{-2}$ to be the lower bound, with the safe upper bound being 100 times this lower value. Thus, in this section, we study the effect of larger values of the exciton-exciton interactions in two different configurations for the polariton system, characterized by detunings $\delta_{C X}=0$ and $\delta_{C X}=-1.08$ in dimensionless units. We consider three different values for the excitonexciton interaction: $g_{X}=2 \mu \mathrm{eV} \mu \mathrm{m}^{-2}, 10 \times 2 \mu \mathrm{eV} \mu \mathrm{m}^{-2}$, $100 \times 2 \mu \mathrm{eV} \mu \mathrm{m}^{-2}$. In Fig. 11, we show the nonlinear parameter $g$ as a function of the normalized external pump power (for zero detuning) and as a function of the anisotropy parameter $\Gamma$ (for finite detuning). We observe that, by increasing the exciton-exciton interaction constant, the system is characterized by larger values of the nonlinear parameter $g$ for the same values of the external pump power (see left panel in Fig. 11). A similar phenomenon appears for finite detuning, where the value of $g$ also increases by increasing $g_{X}$ (see right panel in Fig. 11). However, the difference is not large enough to alter the conclusions presented in the main text.

[1] P. C. Hohenberg and B. I. Halperin, Theory of Dynamic Critical Phenomena, Rev. Mod. Phys. 49, 435 (1977).

[2] S. P. Obukhov, The Problem of Directed Percolation, Phys. A Stat. Mech. Appl. 101, 145 (1980).

[3] H. Hinrichsen, Non-equilibrium Critical Phenomena and Phase Transitions into Absorbing States, Adv. Phys. 49, 815 (2000).
[4] M. Kardar, G. Parisi, and Y. C. Zhang, Dynamic Scaling of Growing Interfaces, Phys. Rev. Lett. 56, 889 (1986).

[5] T. Vicsek, M. Cserz, and V. K. Horváth, Self-Affine Growth of Bacterial Colonies, Phys. A Stat. Mech. Appl. 167, 315 (1990).

[6] J. Wakita, H. Itoh, T. Matsuyama, and M. Matsushita, SelfAffinity for the Growing Interface of Bacterial Colonies, J. Phys. Soc. Jpn. 66, 67 (1997).

[7] M. A. C. Huergo, M. A. Pasquale, A. E. Bolzán, A. J. Arvia, and P.H. González, Morphology and Dynamic Scaling Analysis of Cell Colonies with Linear Growth Fronts, Phys. Rev. E 82, 031903 (2010).

[8] K. A. Takeuchi, M. Sano, T. Sasamoto, and H. Spohn, Growing Interfaces Uncover Universal Fluctuations Behind Scale Invariance, Sci. Rep. 1, 34 (2011).

[9] K. A. Takeuchi and M. Sano, Evidence for GeometryDependent Universal Fluctuations of the Kardar-ParisiZhang Interfaces in Liquid-Crystal Turbulence, J. Stat. Phys. 147, 853 (2012).

[10] F. Ojeda, R. Cuerno, R. Salvarezza, and L. Vázquez, Dynamics of Rough Interfaces in Chemical Vapor Deposition: Experiments and a Model for Silica Films, Phys. Rev. Lett. 84, 3125 (2000).

[11] G. Palasantzas, D. Tsamouras, and J. Th. M. De Hosson, Roughening Aspects of Room Temperature Vapor Deposited Oligomer Thin Films onto Si Substrates, Surf. Sci. 507510, 357 (2002).

[12] R. A. L. Almeida, S. O. Ferreira, T. J. Oliveira, and F. D. A. Aarão Reis, Universal Fluctuations in the Growth of Semiconductor Thin Films, Phys. Rev. B 89, 045309 (2014).

[13] T. Halpin-Healy and G. Palasantzas, Universal Correlators and Distributions as Experimental Signatures of $(2+1)$ Dimensional Kardar-Parisi-Zhang Growth, Europhys. Lett. 105, 50001 (2014).

[14] R. A. L. Almeida, S. O. Ferreira, I. R. B. Ribeiro, and T. J. Oliveira, Temperature Effect on $(2+1)$ Experimental Kardar-Parisi-Zhang Growth, Europhys. Lett. 109, 46003 (2015).

[15] S. G. Alves, C. I. L. de Araujo, and S. C. Ferreira, Hallmarks of the Kardar-Parisi-Zhang Universality Class Elicited by Scanning Probe Microscopy, New J. Phys. 18, 093018 (2016).

[16] J. Maunuksela, M. Myllys, O.-P. Kähkönen, J. Timonen, N. Provatas, M. J. Alava, and T. Ala-Nissila, Kinetic Roughening in Slow Combustion of Paper, Phys. Rev. Lett. 79, 1515 (1997).

[17] M. Myllys, J. Maunuksela, M. Alava, T. Ala-Nissila, J. Merikoski, and J. Timonen, Kinetic Roughening in Slow Combustion of Paper, Phys. Rev. E 64, 036101 (2001).

[18] L. Miettinen, M. Myllys, J. Merikoski, and J. Timonen, Experimental Determination of KPZ Height-Fluctuation Distributions, Eur. Phys. J. B 46, 55 (2005).

[19] I. Carusotto and C. Ciuti, Quantum Fluids of Light, Rev. Mod. Phys. 85, 299 (2013).

[20] H. Deng, H. Haug, and Y. Yamamoto, Exciton-Polariton Bose-Einstein Condensation, Rev. Mod. Phys. 82, 1489 (2010).

[21] E. Altman, L. M. Sieberer, L. Chen, S. Diehl, and J. Toner, Two-Dimensional Superfluidity of Exciton Polaritons Requires Strong Anisotropy, Phys. Rev. X 5, 011017 (2015). 
[22] V. N. Gladilin, K. Ji, and M. Wouters, Spatial Coherence of Weakly Interacting One-Dimensional Nonequilibrium Bosonic Quantum Fluids, Phys. Rev. A 90, 023615 (2014).

[23] K. Ji, V. N. Gladilin, and M. Wouters, Temporal Coherence of One-Dimensional Nonequilibrium Quantum Fluids, Phys. Rev. B 91, 045301 (2015).

[24] L. He, L. M. Sieberer, E. Altman, and S. Diehl, Scaling Properties of One-Dimensional Driven-Dissipative Condensates, Phys. Rev. B 92, 155307 (2015).

[25] J. Keeling, L. M. Sieberer, E. Altman, L. Chen, S. Diehl, and J. Toner, Superfluidity and Phase Correlations of Driven Dissipative Condensates, arXiv:1601.04495.

[26] L. M. Sieberer, M. Buchhold, and S. Diehl, Keldysh Field Theory for Driven Open Quantum Systems, Rep. Prog. Phys. 79, 096001 (2016).

[27] G. Wachtel, L. M. Sieberer, S. Diehl, and E. Altman, Electrodynamic Duality and Vortex Unbinding in DrivenDissipative Condensates, Phys. Rev. B 94, 104520 (2016).

[28] L. M. Sieberer, G. Wachtel, E. Altman, and S. Diehl, Lattice Duality for the Compact Kardar-Parisi-Zhang Equation, Phys. Rev. B 94, 104521 (2016).

[29] L. He, L. M. Sieberer, and S. Diehl, Space-Time Vortex Driven Crossover and Vortex Turbulence Phase Transition in One-Dimensional Driven Open Condensates, Phys. Rev. Lett. 118, 085301 (2017).

[30] L. Chen and J. Toner, Universality for Moving Stripes: A Hydrodynamic Theory of Polar Active Smectics, Phys. Rev. Lett. 111, 088701 (2013).

[31] D. Caputo et al., Topological Order and Equilibrium in a Condensate of Exciton-Polaritons, arXiv:1610.05737.

[32] Y. Sun, P. Wen, Y. Yoon, G. Liu, M. Steger, L. N Pfeiffer, K. West, D. W Snoke, and K. A. Nelson, Bose-Einstein Condensation of Long-Lifetime Polaritons in Thermal Equilibrium, Phys. Rev. Lett. 118, 016602 (2017).

[33] G. Dagvadorj, J. M. Fellows, and S. Matyjaśkiewicz, F. M. Marchetti, I. Carusotto, and M. H. Szymańska, Nonequilibrium Phase Transition in a Two-Dimensional Driven Open Quantum System, Phys. Rev. X 5, 041028 (2015).

[34] D. M. Whittaker, Effects of Polariton-Energy Renormalization in the Microcavity Optical Parametric Oscillator, Phys. Rev. B 71, 115301 (2005).

[35] M. Wouters and I. Carusotto, Parametric Oscillation Threshold of Semiconductor Microcavities in the Strong Coupling Regime, Phys. Rev. B 75, 075332 (2007).

[36] F. M. Marchetti and M. H. Szymańska, Vortices in Polariton OPO Superfluids, in Exciton Polaritons in MicrocavitiesNew Frontiers, edited by V. Timofeev and D. Sanvitto (Springer, Berlin, Heidelberg, 2012), pp. 173-213.

[37] G. Roumpos et al., Power-Law Decay of the Spatial Correlation Function in Exciton-Polariton Condensates, Proc. Natl. Acad. Sci. U.S.A. 109, 6467 (2012).

[38] A. Mitra, S. Takei, Y. B. Kim, and A. J. Millis, Nonequilibrium Quantum Criticality in Open Electronic Systems, Phys. Rev. Lett. 97, 236808 (2006).

[39] E. G. Dalla Torre, E. Demler, T. Giamarchi, and E. Altman, Quantum Critical States and Phase Transitions in the Presence of Non-equilibrium Noise, Nat. Phys. 6, 806 (2010).

[40] S. Diehl, A. Tomadin, A. Micheli, R. Fazio, and P. Zoller, Dynamical Phase Transitions and Instabilities in Open
Atomic Many-Body Systems, Phys. Rev. Lett. 105, 015702 (2010).

[41] S. Diehl, A. Micheli, A. Kantian, B. Kraus, H. P. Buchler, and P. Zoller, Quantum States and Phases in Driven Open Quantum Systems with Cold Atoms, Nat. Phys. 4, 878 (2008).

[42] A. Mitra and T. Giamarchi, Mode-Coupling-Induced Dissipative and Thermal Effects at Long Times after a Quantum Quench, Phys. Rev. Lett. 107, 150602 (2011).

[43] A. Mitra and T. Giamarchi, Thermalization and Dissipation in Out-of-Equilibrium Quantum Systems: A Perturbative Renormalization Group Approach, Phys. Rev. B 85, 075117 (2012).

[44] B. Öztop, M. Bordyuh, Ö.E. Müstecaplığlu, and H.E. Türeci, Excitations of Optically Driven Atomic Condensate in a Cavity: Theory of Photodetection Measurements, New J. Phys. 14, 085011 (2012).

[45] E. G. Dalla Torre, S. Diehl, M. D. Lukin, S. Sachdev, and P. Strack, Keldysh Approach for Nonequilibrium Phase Transitions in Quantum Optics: Beyond the Dicke Model in Optical Cavities, Phys. Rev. A 87, 023831 (2013).

[46] M. Wouters and I. Carusotto, Absence of Long-Range Coherence in the Parametric Emission of Photonic Wires, Phys. Rev. B 74, 245316 (2006).

[47] L. M. Sieberer, S. D. Huber, E. Altman, and S. Diehl, Dynamical Critical Phenomena in Driven-Dissipative Systems, Phys. Rev. Lett. 110, 195301 (2013).

[48] L. M. Sieberer, S. D. Huber, E. Altman, and S. Diehl, Nonequilibrium Functional Renormalization for DrivenDissipative Bose-Einstein Condensation, Phys. Rev. B 89, 134310 (2014).

[49] P. G. Savvidis, J. J. Baumberg, R. M. Stevenson, M. S. Skolnick, D. M. Whittaker, and J.S. Roberts, AngleResonant Stimulated Polariton Amplifier, Phys. Rev. Lett. 84, 1547 (2000).

[50] R. M. Stevenson, V. N. Astratov, M. S. Skolnick, D. M. Whittaker, M. Emam-Ismail, A. I. Tartakovskii, P. G. Savvidis, J.J. Baumberg, and J.S. Roberts, Continuous Wave Observation of Massive Polariton Redistribution by Stimulated Scattering in Semiconductor Microcavities, Phys. Rev. Lett. 85, 3680 (2000).

[51] J. J. Baumberg, P. G. Savvidis, R. M. Stevenson, A. I. Tartakovskii, M. S. Skolnick, D. M. Whittaker, and J. S. Roberts, Parametric Oscillation in a Vertical Microcavity: A Polariton Condensate or Micro-Optical Parametric Oscillation, Phys. Rev. B 62, R16247 (2000).

[52] A. I. Tartakovskii, D. N. Krizhanovskii, D. A. Kurysh, V. D. Kulakovskii, M. S. Skolnick, and J. S. Roberts, Polariton Parametric Scattering Processes in Semiconductor Microcavities Observed in Continuous Wave Experiments, Phys. Rev. B 65, 081308 (2002).

[53] D. Sanvitto et al., Persistent Currents and Quantized Vortices in a Polariton Superfluid, Nat. Phys. 6, 527 (2010).

[54] K. Dunnett and M. H. Szymańska, Keldysh Field Theory for Nonequilibrium Condensation in a Parametrically Pumped Polariton System, Phys. Rev. B 93, 195306 (2016).

[55] L. M. Sieberer, A. Chiocchetta, A. Gambassi, U. C. Täuber, and S. Diehl, Thermodynamic Equilibrium as a Symmetry of the Schwinger-Keldysh Action, Phys. Rev. B 92, 134307 (2015). 
[56] Here and in the following, we are using dimensionless units, measuring time, length, and energy in $2 / \Omega_{R}, \sqrt{\hbar /\left(\Omega_{R} m_{C}\right)}$ and $\hbar \Omega_{R} / 2$, respectively, where $\Omega_{R}$ is the Rabi frequency of the exciton-photon coupling, and $m_{C}$ is the cavity photon effective mass.

[57] L. M. Sieberer, Dissertation, University of Innsbruck, 2015.

[58] A. Altland and B. D. Simons, Condensed Matter Field Theory (Cambridge University Press, Cambridge, England, 2010).

[59] D. E. Wolf, Kinetic Roughening of Vicinal Surfaces, Phys. Rev. Lett. 67, 1783 (1991).

[60] However, even in incoherently pumped polaritons, some degree of anisotropy can be induced by the crystal structure and the splitting of transverse electric and transverse magnetic cavity modes $[19,61]$.

[61] I. A. Shelykh, A. V. Kavokin, Y. G. Rubo, T. C. Liew, and G. Malpuech, Polariton Polarization-Sensitive Phenomena in Planar Semiconductor Microcavities, Semicond. Sci. Technol. 25, 013001 (2010).

[62] We note that $y$ depends on the physics on short scales [information that is not contained in the long-wavelength description, Eq. (9)] but could, in principle, be calculated from the stochastic equations (7).

[63] For $\Gamma=1$, by rescaling the units of length, Eq. (9) can be brought to isotropic form with $D_{x}=D_{y}$ and $\lambda_{x}=\lambda_{y}$.

[64] E. Frey and U.C. Täuber, Two-Loop RenormalizationGroup Analysis of the Burgers-Kardar-Parisi-Zhang Equation, Phys. Rev. E 50, 1024 (1994).

[65] A. Pagnani and G. Parisi, Numerical Estimate of the Kardar-Parisi-Zhang Universality Class in $(2+1)$ Dimensions., Phys. Rev. E 92, 010101 (2015).

[66] L. Canet, H. Chaté, B. Delamotte, and N. Wschebor, Nonperturbative Renormalization Group for the KardarParisi-Zhang Equation, Phys. Rev. Lett. 104, 150601 (2010).

[67] While these estimates were originally derived for isotropic systems, we expect them to remain valid in the WA regime and adapt the expression for $t_{\mathrm{KPZ}}$ to the latter by replacing the isotropic diffusion constant by the geometric mean $\bar{D}=\sqrt{D_{x} D_{y}}$.

[68] F. M. Marchetti, M. H. Szymańska, C. Tejedor, and D. M. Whittaker, Spontaneous and Triggered Vortices in Polariton Optical-Parametric-Oscillator Superfluids, Phys. Rev. Lett. 105, 063902 (2010).

[69] As in the above estimates of the characteristic KPZ scales, we expect to obtain a valid estimate throughout the WA regime by replacing the isotropic diffusion constant and nonlinearity by the geometric averages $\bar{D}$ and $\bar{\lambda}=\sqrt{\lambda_{x} \lambda_{y}}$. Note that $\bar{\xi}_{0}$ is related to the vortex mobility and may differ substantially from $\xi_{0}$ (see Appendix B).

[70] A. D. Rutenberg and A.J. Bray, Phase Ordering of Two-Dimensional XY Systems below the KosterlitzThouless Transition Temperature, Phys. Rev. E 51, R1641 (1995).
[71] M. H. Szymańska, J. Keeling, and P. B. Littlewood, Nonequilibrium Quantum Condensation in an Incoherently Pumped Dissipative System, Phys. Rev. Lett. 96, 230602 (2006).

[72] M. H. Szymańska, J. Keeling, and P. B. Littlewood, MeanField Theory and Fluctuation Spectrum of a Pumped Decaying Bose-Fermi System across the Quantum Condensation Transition, Phys. Rev. B 75, 195331 (2007).

[73] We note, however, that even in the SA regime, the nonlinearities might induce screening of the vortex interaction with a screening length that is shorter than the scale at which $g \approx 0$. It is an interesting question for future research whether this affects the BKT transition.

[74] J. Kasprzak et al., Bose-Einstein Condensation of Exciton Polaritons, Nature (London) 443, 409 (2006).

[75] R. Spano, J. Cuadra, C. Lingg, D. Sanvitto, M. D. Martin, P. R. Eastham, M. van der Poel, J. M. Hvam, and L. Viña, Build up of Off-Diagonal Long-Range Order in Microcavity Exciton-Polaritons across the Parametric Threshold, Opt. Express 21, 10792 (2013).

[76] W. H. Nitsche, N. Y. Kim, G. Roumpos, C. Schneider, M. Kamp, S. Höfling, A. Forchel, and Y. Yamamoto, Algebraic Order and the Berezinskii-Kosterlitz-Thouless Transition in an Exciton-Polariton Gas, Phys. Rev. B 90, 205430 (2014).

[77] K. Dunnett, G. Dagvadorj, A. Zamora, and M. H. Szymanska, Polariton $O P O$ - The Signal Momentum (unpublished).

[78] E. Wertz et al., Spontaneous Formation and Optical Manipulation of Extended Polariton Condensates, Nat. Phys. 6, 860 (2010).

[79] M. Steger, C. Gautham, D. W. Snoke, L. Pfeiffer, and K. West, Slow Reflection and Two-Photon Generation of Microcavity Exciton-Polaritons, Optica 2, 1 (2015).

[80] K. S. Daskalakis, S. A. Maier, R. Murray, and S. KénaCohen, Nonlinear Interactions in an Organic Polariton Condensate, Nat. Mater. 13, 271 (2014).

[81] See Ref. [73]

[82] N. P. Robins, P. A. Altin, J. E. Debs, and J. D. Close, Atom Lasers: Production, Properties and Prospects for Precision Inertial Measurement, Phys. Rep. 529, 265 (2013).

[83] J. S. Douglas, H. Habibian, C. L. Hung, A. V. Gorshkov, H. J. Kimble, and D. E. Chang, Quantum Many-Body Models with Cold Atoms Coupled to Photonic Crystals, Nat. Photonics 9, 326 (2015).

[84] J. D. Hood, A. Goban, A. Asenjo-Garcia, M. Lu, S. Yu, D. E. Chang, and H. J. Kimble, Atom-Atom Interactions around the Band Edge of a Photonic Crystal Waveguide, Proc. Natl. Acad. Sci. U.S.A. 113, 10507 (2016).

[85] I. S. Aranson, S. Scheidl, and V. M. Vinokur, Nonequilibrium Dislocation Dynamics and Instability of Driven Vortex Lattices in Two Dimensions, Phys. Rev. B 58, 14541 (1998).

[86] P. M. Walker, L. Tinkler, D. V. Skryabin, A. Yulin, B. Royall, I. Farrer, D. A. Ritchie, M. S. Skolnick, and D. N. Krizhanovskii, Ultra-Low-Power Hybrid Light-Matter Solitons, Nat. Commun. 6, 8317 (2015). 